\title{
Matching of fracture functions for SIDIS in target fragmentation region
}

\author{
K.B. Chen, ${ }^{a, b}$ J.P. Ma ${ }^{b, c, d}$ and X.B. Tong ${ }^{e, f, b}$ \\ ${ }^{a}$ School of Science, Shandong Jianzhu University, \\ Jinan, Shandong 250101, China \\ ${ }^{b}$ CAS Key Laboratory of Theoretical Physics, Institute of Theoretical Physics, \\ P.O. Box 2735, Chinese Academy of Sciences, Beijing 100190, China \\ ${ }^{c}$ School of Physical Sciences, University of Chinese Academy of Sciences, \\ Beijing 100049, China \\ ${ }^{d}$ School of Physics and Center for High-Energy Physics, Peking University, \\ Beijing 100871, China \\ ${ }^{e}$ School of Science and Engineering, The Chinese University of Hong Kong, Shenzhen, \\ Shenzhen, Guangdong, 518172, China \\ ${ }^{f}$ University of Science and Technology of China, \\ Hefei, Anhui, 230026, China \\ E-mail: chenkaibao19@sdjzu.edu.cn, majp@itp.ac.cn, \\ tongxuanbo@cuhk.edu.cn
}

ABSTRACT: In the target fragmentation region of Semi-Inclusive Deep Inelastic Scattering, the diffractively produced hadron has small transverse momentum. If it is at order of $\Lambda_{\mathrm{QCD}}$, it prevents to make predictions with the standard collinear factorization. However, in this case, differential cross-sections can be predicted by the factorization with fracture functions, diffractive parton distributions. If the transverse momentum is much larger than $\Lambda_{\mathrm{QCD}}$ but much smaller than $Q$ which is the virtuality of the virtual photon, both factorizations apply. In this case, fracture functions can be factorized with collinear parton distributions and fragmentation functions. We study the factorization up to twist-3 level and obtain gauge invariant results. They will be helpful for modeling fracture functions and useful for resummation of large logarithm of the transverse momentum appearing in collinear factorization.

Keywords: Deep Inelastic Scattering (Phenomenology), QCD Phenomenology

ArXIV EPRINT: 2108.13582 


\section{Contents}

1 Introduction 1

2 Notations and matching of twist-2 fracture functions 2

2.1 Definitions of fracture functions and SIDIS in target fragmentation region 2

2.2 Matching of $F_{q}$ and $\Delta F_{q}$

$\begin{array}{lll}2.3 & \text { Definitions of twist-3 parton distributions } & 7\end{array}$

3 Matching of $F_{q T} \quad 9$

$\begin{array}{lll}4 & \text { Matching of } \Delta F_{q T} & 14\end{array}$

$\begin{array}{lll}5 & \text { Summary } & 20\end{array}$

\section{Introduction}

Semi-Inclusive DIS(SIDIS) have three different kinematical regions in which different QCD factorizations are used. These regions are classified according to the momentum of the observed hadron in the final state. In the region where the transverse momentum is large, the production rate can be predicted with standard QCD collinear factorization. In the region where the observed hadron moves in the forward region of the virtual photon with a small transverse momentum, one can use Transverse-Momentum-Dependent QCD factorization [1-4], which involves TMD parton distributions and TMD parton fragmentation functions. The third region is called as target fragmentation region, where the observed hadron moves in the forward region of the initial hadron with a small transverse momentum $k_{\perp}$. In this region, the standard collinear factorization fails because of that the perturbative part in the factorization becomes divergent as powers of $\ln k_{\perp}$.

Experimentally, the production in the target fragmentation region has been observed in HERA experiment [5]. It has been suggested in [6] that the production rate can be factorized with fracture functions. An one-loop study of SIDIS in [7] shows that the factorization holds at the order. One can prove the factorization with fracture functions at all orders, as discussed in [8,9]. For the production of one hadron combined with a lepton-pair in hadron collisions, factorizations with fracture functions have been shown to hold at one-loop level in $[10,11]$, where the produced hadron is in the forward- or backward regions. However, the factorization for hadron collisions can be failed as discussed in [12]. Fracture functions, also called as diffractive parton distributions, are parton distributions of an initial hadron with one diffractively produced particle observed in the final state. Currently, most information about fracture functions comes from analysis of HERA data, e.g., in $[13,14]$. The production in target fragmentation region can be studied with current experiments at JLab and planned experiments at Eic [15] and EicC [16, 17]. Hence, more information of fracture functions will be available. 
It is noted that the factorization in the target fragmentation region holds for $Q \gg k_{\perp}$ where $Q$ is the square root of the momentum transfer of the virtual photon of SIDIS. In the case of $Q \gg k_{\perp} \gg \Lambda_{\mathrm{QCD}}$, one can also use the collinear factorization to factorize the differential cross-sections with collinear parton distributions and fragmentation functions. Therefore, for $k_{\perp} \gg \Lambda_{\mathrm{QCD}}$, fracture functions in SIDIS can be factorized with collinear parton distributions and fragmentation functions. In this paper, we study the factorization up to twist-3. The matching of twist-2 fracture functions is straightforward, while the matching of twist-3 fracture functions is nontrivial in the sense that one has to keep the gauge invariance. Here we will mainly study the factorization or matching at the level of twist-3.

Fracture functions at twist-2 relevant to SIDIS have been defined in [8], where their asymptotic behavior has been derived for the region where the momentum fraction of the struck parton approaches its maximal value. In [18] TMD quark fracture functions have been classified for a polarized spin 1/2 hadron in the initial state. An one-loop matching of various twist-2 fracture functions for the process where the production of a lepton pair with large invariant mass in hadron collisions associated with a diffractively produced photon instead of a hadron in different kinematical regions, has been studied in [19].

In the target fragmentation region with $Q \gg k_{\perp}$ SIDIS can be conveniently described with fracture functions. If one uses collinear factorization in the region of $Q \gg k_{\perp} \gg \Lambda_{\mathrm{QCD}}$, then there will be terms with large log's like $\ln Q / k_{\perp}$ in perturbative coefficient functions. Using the factorization with fracture functions and the matching studied here, these terms with large log's can be re-summed with helps of evolution equations. In collinear factorization, twist-3 effects involve in general twist-3 parton distributions and twist-3 parton fragmentation functions. It is interesting to notice that the latter is absent in the matching of twist-3 studied here. The reason for this is helicity conservation in QCD as we will show.

The content of our paper is: in section 2. we introduce our notations and definitions of relevant parton distributions, fragmentation functions and fracture functions. The results of matching of twist-2 fracture functions are also given in this section. In section 3. we derive the matching of the twist-3 fracture function which is relevant to single transverse-spin asymmetry. In section 4 . the matching of the twist-3 fracture function responsible to a double-spin asymmetry is given. Section 5 is our summary.

\section{Notations and matching of twist-2 fracture functions}

We will use the light-cone coordinate system, in which a vector $a^{\mu}$ is expressed as $a^{\mu}=$ $\left(a^{+}, a^{-}, \vec{a}_{\perp}\right)=\left(\left(a^{0}+a^{3}\right) / \sqrt{2},\left(a^{0}-a^{3}\right) / \sqrt{2}, a^{1}, a^{2}\right)$ and $a_{\perp}^{2}=\left(a^{1}\right)^{2}+\left(a^{2}\right)^{2}$. We introduce two light cone vectors $n^{\mu}=(0,1,0,0)$ and $l^{\mu}=(1,0,0,0)$. The transverse metric is given by $g_{\perp}^{\mu \nu}=g^{\mu \nu}-n^{\mu} l^{\nu}-n^{\nu} l^{\mu}$. We will also need the transverse antisymmetric tensor which is given by $\epsilon_{\perp}^{\mu \nu}=\epsilon^{\alpha \beta \mu \nu} l_{\alpha} n_{\beta}$ with $\epsilon_{\perp}^{12}=1$. Throughout of the work, we will use Feynman gauge.

\subsection{Definitions of fracture functions and SIDIS in target fragmentation region}

We consider an initial hadron $h_{A}$ with the momentum $P^{\mu}=\left(P^{+}, P^{-}, 0,0\right)$ and another hadron in the final state with the momentum $k^{\mu}=\left(k^{+}, k^{-}, k_{\perp}^{1}, k_{\perp}^{2}\right)$ with $k^{+}=\xi P^{+}$. The 
transverse momentum $k_{\perp}$ is small in comparison with $k^{+}$. The quark fracture functions or quark diffractive parton distributions can be defined with the density matrix:

$$
\mathcal{M}_{F i j}\left(x, \xi, k_{\perp}\right)=\int \frac{d \lambda}{2 \pi} e^{-i x P^{+} \lambda} \sum_{X}\left\langle h_{A}\left|\left[\bar{q}(\lambda n) \mathcal{L}_{n}^{\dagger}(\lambda n)\right]_{j}\right| X h\right\rangle\left\langle h X\left|\left[\mathcal{L}_{n}(0) q(0)\right]_{i}\right| h_{A}\right\rangle,
$$

with $i j$ stand for Dirac- and color indices. Here the transverse momentum of the struck quark is integrated over. To make the density matrix gauge-invariant, the gauge links along the $n$-direction are implemented:

$$
\mathcal{L}_{n}(x)=\mathrm{P} \exp \left\{-i g_{s} \int_{0}^{\infty} d \lambda G^{+}(\lambda n+x)\right\} .
$$

The decomposition of the density matrix has been done in [18]. We consider the case that the initial hadron is a spin- $1 / 2$ particle and the polarization of the final hadron is summed if it has nonzero spin. The spin vector of the initial hadron can be decomposed as:

$$
s^{\mu}=s_{L} \frac{l^{\mu} P \cdot n-n^{\mu} P \cdot l}{m_{A}}+s_{\perp}^{\mu}, \quad P^{2}=m_{A}^{2}
$$

with $s_{L}$ as the helicity. Neglecting terms beyond twist-3, the density matrix can be decomposed as:

$$
\begin{aligned}
\mathcal{M}_{F i j}(x, k)= & \frac{1}{2 N_{c}}\left(\gamma^{-}\right)_{i j}\left[F_{q}\left(x, \xi, k_{\perp}\right)+\epsilon_{\perp}^{\mu \nu} k_{\perp \mu} s_{\perp \nu} \frac{1}{m_{A}} F_{q T}\left(x, \xi, k_{\perp}\right)\right] \\
& +\frac{1}{2 N_{c}}\left(\gamma_{5} \gamma^{-}\right)_{i j}\left[s_{L} \Delta F_{q}\left(x, \xi, k_{\perp}\right)+k_{\perp} \cdot s_{\perp} \frac{1}{m_{A}} \Delta F_{q T}\left(x, \xi, k_{\perp}\right)\right]+\cdots
\end{aligned}
$$

where $\cdots$ denote those distributions defined with chirality-odd operators. These distributions will not contribute to SIDIS in target fragmentation region. The function $F_{q}$ and $\Delta F_{q}$ are of twist-2, while $F_{q T}$ and $\Delta F_{q T}$ are of twist-3. We neglect here the contributions beyond twist-3.

We consider the SIDIS process:

$$
e\left(k_{e}, \lambda_{e}\right)+h_{A}(P, s) \rightarrow e\left(k_{e}^{\prime}\right)+h(k)+X
$$

where the initial hadron is of spin- $1 / 2$ with the spin vector $s$. The initial electron can be polarized with the helicity $\lambda_{e}$. We assume that the polarization of the hadron in the final state is not observed. At leading order of QED, there is an exchange of one virtual photon with the momentum $q=k_{e}-k_{e}^{\prime}$ between the electron and the initial hadron. The relevant hadronic tensor is:

$$
W^{\mu \nu}=\sum_{X} \int \frac{d^{4} x}{(2 \pi)^{4}} e^{i q \cdot x}\left\langle h_{A}\left|J^{\mu}(x)\right| h X\right\rangle\left\langle X h\left|J^{\nu}(0)\right| h_{A}\right\rangle .
$$

The standard variables for SIDIS are:

$$
Q^{2}=-q^{2}, \quad x_{B}=\frac{Q^{2}}{2 P \cdot q}, \quad y=\frac{P \cdot q}{P \cdot k_{e}}, \quad z_{h}=\frac{P \cdot k}{P \cdot q} .
$$




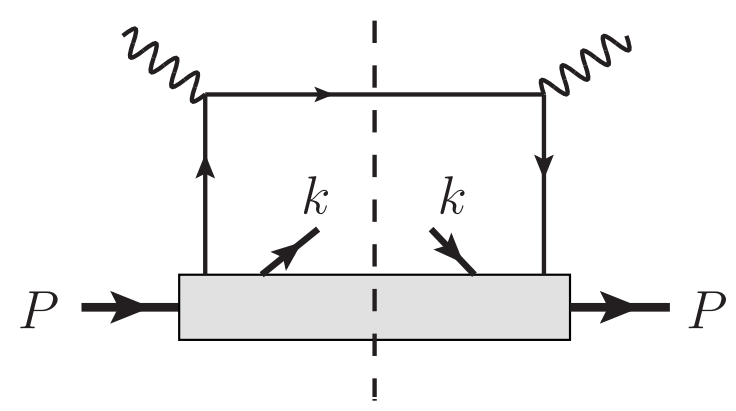

Figure 1. Diagram for SIDIS in target fragmentation region.

The masses of hadrons and leptons are neglected. We take a frame in which the momenta of the initial hadron and the virtual photon are:

$$
P^{\mu} \approx\left(P^{+}, 0,0,0\right), \quad q^{\mu}=\left(q^{+}, q^{-}, 0,0\right),
$$

i.e., the hadron moves in the $z$-direction, and the virtual photon moves in the $-z$-direction. We consider the case in which the transverse momentum $k_{\perp}$ is small with $k_{\perp}^{2} \ll Q^{2}$. In the region $z_{h} \sim \mathcal{O}(1)$, the produced hadron moves almost in the forward region of the virtual photon. This region is called as current fragmentation region. Transverse-MomentumDependent(TMD) factorization applies in this region. In the region of $z_{h} \ll 1$, the produced hadron moves almost in the forward region of the initial hadron. This region is called as target fragmentation region. In this region the hadronic tensor can be factorized with the above introduced fracture functions.

In the target fragmentation region, we write the momentum of the produced hadron as:

$$
k^{\mu}=\xi P^{+} l^{\mu}+k^{-} n^{\mu}+k_{\perp}^{\mu}, \quad k^{-}=\frac{k_{\perp}^{2}}{2 \xi P^{+}}, \quad z_{h}=\frac{x_{B} k_{\perp}^{2}}{\xi Q^{2}} .
$$

We consider the experimental situation in which the initial hadron is polarized and the transverse part $s_{\perp}^{\mu}$ of the spin vector $s^{\mu}$ is nonzero. The incoming- and outgoing lepton span the so-called lepton plane. In our frame specified in the above the azimuthal angle between the spin vector $s_{\perp}$ and the lepton plane is denoted $\phi_{s}$. Similarly, one defines the azimuthal angle $\phi_{h}$ for the produced hadron. The azimuthal angle of the outgoing lepton around the lepton beam with respect to the spin vector is denoted $\psi$. In the kinematical region of SIDIS with large $Q^{2}$, one has $\psi \approx \phi_{s}$ [20]. With this specification the differential cross-section is given by [20, 21]:

$$
\frac{d \sigma}{d x_{B} d y d \xi d \psi d^{2} k_{\perp}}=\frac{\alpha^{2} y}{4 \xi Q^{4}} L_{\mu \nu} W^{\mu \nu},
$$

where $\alpha$ is the fine structure constant. At the leading order, the hadronic tensor receives the contribution from figure 1 where the gray box represents the density matrix in eq. (2.1). From figure 1 the tensor is given by:

$$
W^{\mu \nu}(P, q, k)=\frac{e_{q}^{2}}{(2 \pi)^{4}} \int d\left(x P^{+}\right)(2 \pi) \delta\left((x P+q)^{2}\right)\left(\gamma^{\mu} \gamma \cdot(x P+q) \gamma^{\nu}\right)_{j i} \mathcal{M}_{F i j}\left(x, \xi, k_{\perp}\right)+\cdots
$$


where $\cdots$ are power-suppressed terms. With the fracture functions in eq. (2.4) we can derive the hadronic tensor at the order. With the leptonic tensor at the leading order of QED, we have the differential cross-section in the target fragmentation region expressed with fracture functions as [18]:

$$
\begin{aligned}
& \frac{d \sigma}{d x_{B} d y d \xi d \psi d^{2} k_{\perp}} \\
& =\frac{\alpha^{2}}{2(2 \pi)^{3} \xi y Q^{2}}\left[\left(2-2 y+y^{2}\right)\left(F_{q}\left(x_{B}, \xi, k_{\perp}\right)+\frac{\left|k_{\perp}\right|\left|s_{\perp}\right|}{m_{A}} \sin \left(\phi_{s}-\phi_{h}\right) F_{q T}\left(x_{B}, \xi, k_{\perp}\right)\right)\right. \\
& \left.\quad+\lambda_{e} y(2-y)\left(s_{L} \Delta F_{q}\left(x_{B}, \xi, k_{\perp}\right)-\frac{\left|k_{\perp}\right|\left|s_{\perp}\right|}{m_{A}} \cos \left(\phi_{s}-\phi_{h}\right) \Delta F_{q T}\left(x_{B}, \xi, k_{\perp}\right)\right)\right],
\end{aligned}
$$

with

$$
s_{\perp}^{\mu}=\left|s_{\perp}\right|\left(\cos \phi_{s}, \sin \phi_{s}\right), \quad k_{\perp}^{\mu}=\left|k_{\perp}\right|\left(\cos \phi_{h}, \sin \phi_{h}\right) .
$$

We notice that the factorization in eq. (2.12) holds in fact for the case with $k_{\perp} \ll Q$. In the case of $Q \gg k_{\perp} \gg \Lambda_{\mathrm{QCD}}$, collinear factorization also holds. Therefore, fracture functions in this case can be matched to collinear parton distributions. The function $F_{q}$ and $\Delta F_{q}$ are matched to twist-2 parton distributions and fragmentation functions. The function $F_{q T}$ and $\Delta F_{q T}$ are matched to twist-3 parton distributions and twist-2 parton fragmentation functions. It is interesting to note that there is no contribution involving twist-3 parton fragmentation functions because of helicity conservation. This will be explained later in detail.

\subsection{Matching of $F_{q}$ and $\Delta F_{q}$}

The fracture function $F_{q}$ and $\Delta F_{q}$ can be matched to twist-2 parton distributions and twist-2 parton fragmentation functions, in the case of $k_{\perp} \gg \Lambda_{\mathrm{QCD}}$. These twist-2 quarkand gluon distributions can be defined as:

$$
\begin{aligned}
& \int \frac{d y}{2 \pi} e^{-i y x P^{+}}\left\langle h_{A}\left|\left(\bar{\psi}(y n) \mathcal{L}_{n}^{\dagger}(y n)\right)_{\beta}\left(\mathcal{L}_{n}(0) \psi(0)\right)_{\alpha}\right| h_{A}\right\rangle \\
& =\frac{1}{2 N_{c}}\left[q(x) \gamma^{-}+s_{L} \Delta q(x) \gamma_{5} \gamma^{-}+h_{1}(x) \gamma_{5} \gamma \cdot s_{\perp} \gamma^{-}\right]_{\alpha \beta}+\cdots, \\
& \frac{1}{x P^{+}} \int \frac{d \lambda}{2 \pi} e^{-i x \lambda P^{+}}\left\langle h_{A}\left|\left(G^{+\mu}(\lambda n) \mathcal{L}_{n}^{\dagger}(\lambda n)\right)^{a}\left(\mathcal{L}_{n}(0) G^{+\nu}(0)\right)^{a}\right| h_{A}\right\rangle \\
& \quad=-\frac{1}{2} g_{\perp}^{\mu \nu} g(x)-\frac{i}{2} \epsilon_{\perp}^{\mu \nu} s_{L} \Delta g(x)+\cdots .
\end{aligned}
$$

In the above, $q(x)$ is the spin-averaged quark distribution function, $\Delta q$ is the longitudinally polarized quark distribution, $h_{1}$ is the quark transversity distribution. $\cdots$ stand for twist-3 or higher twists. At twist-2 there are two gluon distributions. $g(x)$ is the spin-averaged gluon distribution function, $\Delta g$ is the longitudinally polarized gluon distribution. At twist-2 the relevant parton fragmentation functions of an unpolarized hadron are defined as:

$$
\begin{aligned}
& d_{\bar{q}}(z)=\frac{z}{2 N_{c}} \int \frac{d \lambda}{4 \pi} e^{-i \lambda k^{+} / z}\left\langle 0\left|\bar{\psi}(0) \mathcal{L}_{n}^{\dagger}(0) \gamma^{+}\right| h, X\right\rangle\left\langle X, h\left|\mathcal{L}_{n}(\lambda n) \psi(\lambda n)\right| 0\right\rangle, \\
& d_{g}(z)=\frac{z}{\left(N_{c}^{2}-1\right) k^{+}} \int \frac{d \lambda}{4 \pi} e^{-i \lambda k^{+} / z}\left\langle 0\left|G^{a,+\mu}(0) \mathcal{L}_{n}^{\dagger}(0)\right| h, X\right\rangle\left\langle X, h\left|\mathcal{L}_{n}(\lambda n) G^{a,+}{ }_{\mu}(\lambda n)\right| 0\right\rangle,
\end{aligned}
$$



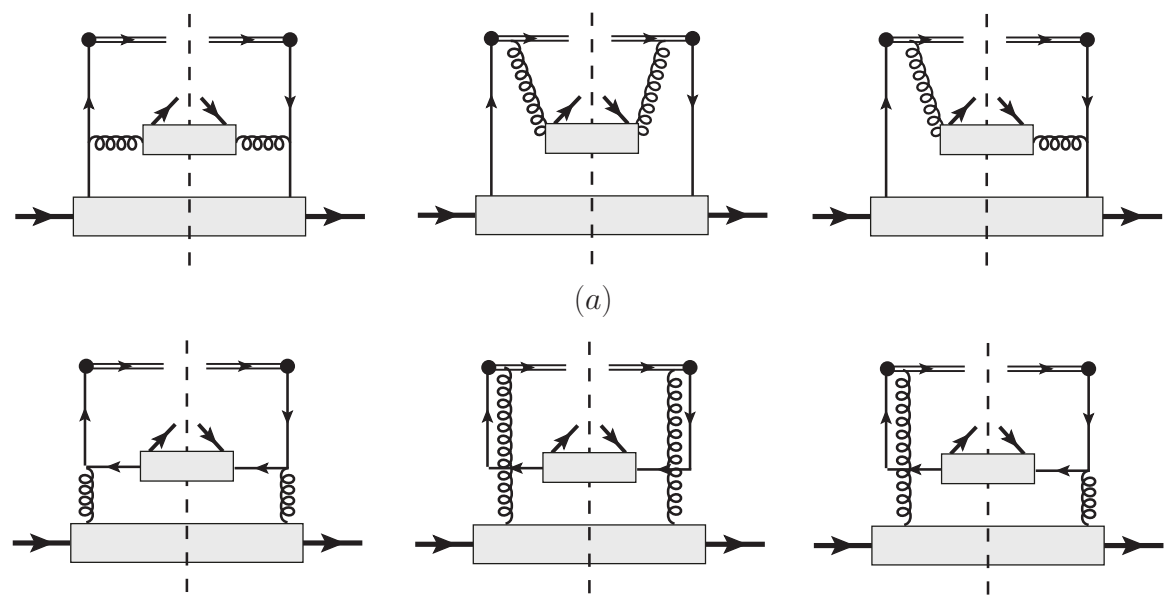

$(b)$

Figure 2. Diagrams for the matching of quark fracture functions to parton distribution functions. (a): contributions from quark parton distributions. (b): contributions from gluon distributions.

where $d_{\bar{q}}$ is the anti-quark fragmentation function, $d_{g}$ is the gluon fragmentation function. The observed hadron in the final state is with the momentum $k^{\mu}=\left(k^{+}, 0,0,0\right)$, where its mass is neglected.

With the twist-2 parton distributions and fragmentation functions, one can easily calculate the fracture functions $F_{q}$ and $\Delta F_{q}$ at tree-level, where nonperturbative effects are represented by these distributions and functions. The contributions to $F_{q}$ and $\Delta F_{q}$ are given by figure 2. Calculations of these diagrams are rather standard. Therefore, we give our twist-2 results directly. More technical details will be explained in subsequent sections. From figure 2 we obtain the fracture functions $F_{q}$ and $\Delta F_{q}$ :

$$
\begin{gathered}
F_{q}\left(x, \xi, k_{\perp}\right)=g_{s}^{2} \frac{1}{k_{\perp}^{2}} \int \frac{d z}{z^{2}}\left[2 C_{F} d_{g}(z) q(y) \frac{z^{2}}{y^{2}}\left(x^{2}+y^{2}\right)+d_{\bar{q}}(z) g(y) \frac{\xi}{z y^{3}}\left(z^{2} x^{2}+\xi^{2}\right)\right], \\
\Delta F_{q}\left(x, \xi, k_{\perp}\right)=g_{s}^{2} \frac{1}{k_{\perp}^{2}} \int \frac{d z}{z^{2}}\left[2 C_{F} d_{g}(z) \Delta q(y) \frac{z^{2}}{y^{2}}\left(x^{2}+y^{2}\right)+d_{\bar{q}}(z) \Delta g(y) \frac{\xi}{y^{2}}(x z-\xi)\right],
\end{gathered}
$$

with

$$
y=x+\frac{\xi}{z} .
$$

We notice that for $k_{\perp} \gg \Lambda_{\mathrm{QCD}}$, the twist-2 fracture functions behave like $1 / k_{\perp}^{2}$.

The other two fracture functions $F_{q T}$ and $\Delta F_{q T}$ are for the initial hadron which is transversely polarized. At first look, the twist- 2 transversity distribution $h_{1}$, which is chirality-odd, can give contributions to these functions from figure 2, where twist-3 parton fragmentation functions are involved. At the order we consider, helicity or chirality is conserved in the amplitude of the upper part in figure 1 . Therefore, contributions involve chirality-odd parton distributions are absent. Hence, the two fracture functions only receive contributions where twist-3 parton distributions and twist-2 parton fragmentation functions are involved. Before we turn to the matching of these two functions, we give definitions of twist-3 parton distributions in the next subsection. 


\subsection{Definitions of twist-3 parton distributions}

From the quark density matrix one can define a set of three twist-3 parton distributions with one transverse derivative [22]:

$$
\begin{aligned}
q_{T}(x) s_{\perp}^{\mu} & =P^{+} \int \frac{d \lambda}{4 \pi} e^{-i x \lambda P^{+}}\left\langle h_{A}\left|\bar{\psi}(\lambda n) \mathcal{L}_{n}^{\dagger}(\lambda n) \gamma_{\perp}^{\mu} \gamma_{5} \mathcal{L}_{n}(0) \psi(0)\right| h_{A}\right\rangle, \\
-i q_{\partial}(x) s_{\perp}^{\mu} & =\int \frac{d \lambda}{4 \pi} e^{-i x \lambda P^{+}}\left\langle h_{A}\left|\bar{\psi}(\lambda n) \mathcal{L}_{n}^{\dagger}(\lambda n) \gamma^{+} \gamma_{5} \partial_{\perp}^{\mu}\left(\mathcal{L}_{n} \psi\right)(0)\right| h_{A}\right\rangle, \\
-i q_{\partial}^{\prime}(x) \tilde{s}_{\perp}^{\mu} & =\int \frac{d \lambda}{4 \pi} e^{-i x \lambda P^{+}}\left\langle h_{A}\left|\bar{\psi}(\lambda n) \mathcal{L}_{n}^{\dagger}(\lambda n) \gamma^{+} \partial_{\perp}^{\mu}\left(\mathcal{L}_{n} \psi\right)(0)\right| h_{A}\right\rangle,
\end{aligned}
$$

with $\tilde{s}^{\mu}=\epsilon_{\perp}^{\mu \nu} s_{\perp \nu}$. The three distributions are real.

Another set of twist-3 distributions can be defined with a pair of quark fields with one gluon field strength operator. They are given by the matrix:

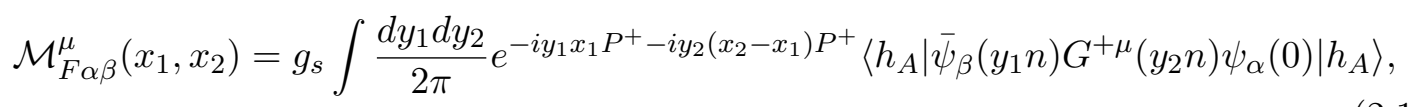

where we have suppressed the gauge links for short notations. The matrix can be decomposed into

$$
\begin{aligned}
\mathcal{M}_{F}^{\mu}\left(x_{1}, x_{2}\right)= & \frac{1}{2}\left[T_{F}\left(x_{1}, x_{2}\right) \tilde{s}_{\perp}^{\mu} \gamma^{-}+T_{\Delta}\left(x_{1}, x_{2}\right) i s_{\perp}^{\mu} \gamma_{5} \gamma^{-}\right] \\
& +\frac{1}{4}\left[s_{L} \tilde{T}_{\Delta, F}\left(x_{1}, x_{2}\right) i \gamma_{5} \gamma_{\perp}^{\mu} \gamma^{-}+\tilde{T}_{F}\left(x_{1}, x_{2}\right) i \gamma_{\perp}^{\mu} \gamma^{-}\right] .
\end{aligned}
$$

Properties of these twist-3 parton distributions have been studied in [23-30]. The first two have been introduced in the study of single transverse-spin asymmetries. The last two are chirality-odd. They will not give contributions in the matching because of helicity conservation. The chirality-even parton distributions satisfy:

$$
T_{F}\left(x_{1}, x_{2}\right)=T_{F}\left(x_{2}, x_{1}\right), \quad T_{\Delta}\left(x_{1}, x_{2}\right)=-T_{\Delta}\left(x_{2}, x_{1}\right) .
$$

Corresponding to the two distributions in eq. (2.20) one can define additionally two twist-3 distributions by replacing the field strength tensor $g_{s} G^{+\mu}(x)$ with $P^{+} D_{\perp}^{\mu}(x)$, where $D^{\mu}(x)$ is given by $D^{\mu}(x)=\partial_{\mu}+i g_{s} G^{\mu}(x)$. These two functions will not appear in our calculation. In fact they can be expressed with the distributions given in eqs. (2.18), (2.20) as shown in [31].

The three twist-3 distributions in eq. (2.18) and the two twist-3 chirality-even distributions in eq. (2.20) are not independent. One can show:

$\frac{1}{2 \pi} \int d x_{1} P \frac{1}{x_{1}-x_{2}}\left[T_{F}\left(x_{1}, x_{2}\right)-T_{\Delta}\left(x_{1}, x_{2}\right)\right]=-x_{2} q_{T}\left(x_{2}\right)+q_{\partial}\left(x_{2}\right), \quad T_{F}(x, x)=-2 q_{\partial}^{\prime}(x)$,

where $P$ stands for the principle-value prescription. The first relation has been derived in [32]. The second relation is derived in [22]. It should be emphasized that the second relation is for SIDIS, for $q_{\partial}^{\prime}$ defined with future pointing gauge links. The distribution $q_{\partial}^{\prime}(x)$ in Drell-Yan processes is defined with gauge links pointing to the past. With the 
symmetries of time-reversal and parity one can show that there is a sign-difference between the two distributions.

There are twist-3 gluon distributions defined only with gluon fields. One can first define three density matrices:

$$
\begin{aligned}
M_{\partial}^{\mu \nu \rho}(x)= & \frac{1}{x P^{+}} \int \frac{d \lambda}{2 \pi} e^{-i x \lambda P^{+}}\left\langle h_{A}\left|\left(G^{+\mu}(\lambda n) \mathcal{L}_{n}^{\dagger}(\lambda n)\right)^{a} \partial_{\perp}^{\rho}\left(\mathcal{L}_{n}(0) G^{+\nu}(0)\right)^{a}\right| h_{A}\right\rangle \\
M_{D}^{\mu \nu \rho}\left(x_{1}, x_{2}\right)= & \int \frac{d \lambda_{1}}{2 \pi} \frac{d \lambda_{2}}{2 \pi} e^{-i \lambda_{1} x_{1} P^{+}-i \lambda_{2}\left(x_{2}-x_{1}\right) P^{+}} \\
& \left\langle h_{A}\left|\left(G^{+\mu}\left(\lambda_{1} n\right) \mathcal{L}\left(\lambda_{1} n\right)\right)_{a}\left(\mathcal{L}\left(\lambda_{2} n\right) D_{\perp}^{\rho}\left(\lambda_{2} n\right) \mathcal{L}^{\dagger}\left(\lambda_{2} n\right)\right)_{a b}\left(\mathcal{L}(0) G^{+\nu}(0)\right)_{b}\right| h_{A}\right\rangle, \\
M_{F}^{\mu \nu \rho}\left(x_{1}, x_{2}\right)= & -\frac{g_{s}}{P^{+}} \int \frac{d \lambda_{1}}{2 \pi} \frac{d \lambda_{2}}{2 \pi} e^{-i \lambda_{1} x_{1} P^{+}-i \lambda_{2}\left(x_{2}-x_{1}\right) P^{+}} \\
& \left\langle h_{A}\left|\left(G^{+\mu}\left(\lambda_{1} n\right) \mathcal{L}\left(\lambda_{1} n\right)\right)_{a}\left(\mathcal{L}\left(\lambda_{2} n\right) G^{+\rho}\left(\lambda_{2} n\right) \mathcal{L}^{\dagger}\left(\lambda_{2} n\right)\right)_{a b}\left(\mathcal{L}(0) G^{+\nu}(0)\right)_{b}\right| h_{A}\right\rangle,
\end{aligned}
$$

where the field strength tensor $G^{+\rho}\left(\lambda_{2} n\right)$ in the last line and the covariant derivative $D^{\rho}\left(\lambda_{2} n\right)$ are in the adjoint representation. The three matrices are not independent. They are related through the relation:

$$
M_{D}^{\mu \nu \rho}\left(x_{1}, x_{2}\right)=x_{1} \delta\left(x_{1}-x_{2}\right) M_{\partial}^{\mu \nu \rho}\left(x_{1}\right)-\frac{1}{x_{2}-x_{1}+i \varepsilon} M_{F}^{\mu \nu \rho}\left(x_{1}, x_{2}\right) .
$$

Besides the three matrices at twist-3 one can also find an additional twist-3 matrices conveniently in the light-cone gauge $G^{+}=0$. In this gauge, one can introduce

$$
\begin{gathered}
\frac{i^{3} g_{s}}{P^{+}} \int \frac{d \lambda_{1}}{2 \pi} \frac{d \lambda_{2}}{2 \pi} e^{i \lambda_{1} x_{1} P^{+}+i \lambda_{2}\left(x_{2}-x_{1}\right) P^{+}}\left\langle h_{A}\left|G^{a,+\alpha}\left(\lambda_{1} n\right) G^{c,+\gamma}\left(\lambda_{2} n\right) G^{b,+\beta}(0)\right| h_{A}\right\rangle \\
=\frac{N_{c}}{\left(N_{c}^{2}-1\right)\left(N_{c}^{2}-4\right)} d^{a b c} O^{\alpha \beta \gamma}\left(x_{1}, x_{2}\right)-\frac{i}{N_{c}\left(N_{c}^{2}-1\right)} f^{a b c} N^{\alpha \beta \gamma}\left(x_{1}, x_{2}\right),
\end{gathered}
$$

where all indices $\alpha, \beta$ and $\gamma$ are transverse. One can identify that:

$$
M_{F}^{\mu \nu \rho}\left(x_{1}, x_{2}\right)=i N^{\mu \nu \rho}\left(-x_{1},-x_{2}\right) .
$$

Therefore, there are two twist-3 matrices built with three gluon field strength tensor operators. From Bose-symmetry and covariance the two tensors take the form [33-35]:

$$
\begin{aligned}
& O^{\alpha \beta \gamma}\left(x_{1}, x_{2}\right)=-2 i\left[O\left(x_{1}, x_{2}\right) g_{\perp}^{\alpha \beta} \tilde{s}_{\perp}^{\gamma}+O\left(x_{2}, x_{2}-x_{1}\right) g_{\perp}^{\beta \gamma} \tilde{s}_{\perp}^{\alpha}+O\left(x_{1}, x_{1}-x_{2}\right) g_{\perp}^{\gamma \alpha} \tilde{s}_{\perp}^{\beta}\right], \\
& N^{\alpha \beta \gamma}\left(x_{1}, x_{2}\right)=-2 i\left[N\left(x_{1}, x_{2}\right) g_{\perp}^{\alpha \beta} \tilde{s}_{\perp}^{\gamma}-N\left(x_{2}, x_{2}-x_{1}\right) g_{\perp}^{\beta \gamma} \tilde{s}_{\perp}^{\alpha}-N\left(x_{1}, x_{1}-x_{2}\right) g_{\perp}^{\gamma \alpha} \tilde{s}_{\perp}^{\beta}\right],
\end{aligned}
$$

with the properties of the functions $O$ and $N$

$$
\begin{array}{ll}
O\left(x_{1}, x_{2}\right)=O\left(x_{2}, x_{1}\right), & O\left(x_{1}, x_{2}\right)=O\left(-x_{1},-x_{2}\right), \\
N\left(x_{1}, x_{2}\right)=N\left(x_{2}, x_{1}\right), & N\left(x_{1}, x_{2}\right)=-N\left(-x_{1},-x_{2}\right) .
\end{array}
$$


The matrix $M_{\partial}^{\mu \nu \rho}(x)$ can be parametrized with constraints of symmetries as:

$$
M_{\partial}^{\mu \nu \rho}(x)=i g_{\perp}^{\mu \nu} \tilde{s}_{\perp}^{\rho} g_{\partial}(x)+i\left(g_{\perp}^{\mu \rho} \tilde{s}_{\perp}^{\nu}+g_{\perp}^{\nu \rho} \tilde{s}_{\perp}^{\mu}\right) g_{\partial}^{\prime}(x)
$$

with

$$
g_{\partial}(x)=g_{\partial}(-x), \quad g_{\partial}^{\prime}(x)=g_{\partial}^{\prime}(-x) .
$$

The two functions $g_{\partial}$ and $g_{\partial}^{\prime}$ are real. From the relation in eq. (2.24) one has:

$$
x g_{\partial}(x)=2 \pi N(x, x), \quad x g_{\partial}^{\prime}(x)=-2 \pi N(x, 0) .
$$

Therefore, all twist-3 gluon distributions are determined by $N\left(x_{1}, x_{2}\right)$ and $O\left(x_{1}, x_{2}\right)$.

In our notations, all twist-3 parton distributions have the dimension 1 in mass and are proportional to $\Lambda_{\mathrm{QCD}}$.

\section{$3 \quad$ Matching of $F_{q T}$}

$F_{q T}$ describes the single transverse-spin asymmetry of SIDIS in target fragmentation region. It is well-known that such an asymmetry is a T-odd effect which requires nonzero absorptive parts in the scattering amplitude. In the case of $k_{\perp} \gg \Lambda_{\mathrm{QCD}}$ the asymmetry is a twist-3 effect in the collinear factorization [23-27]. In this case, $F_{q T}$ can be matched with twist3 parton distributions and twist-2 parton fragmentation functions. Because of helicity conservation, the contribution involving the twist-2 quark distribution $h_{1}$ with twist-3 parton fragmentation functions is absent as discussed before.

At the order considered, there is no contributions from figure 2 because of the absence of the required absorptive part. The contributions are given by figure 3 , figure 4 and figure 5 , where the diagrams are given in terms of amplitude. The contributions are obtained from the interference of the amplitudes of figure $3 \mathrm{a}$, figure 4 and figure 5 with those of figure $3 \mathrm{~b}$. There is one propagator in figure $3 \mathrm{a}$, figure 4 and figure 5 with a short bar. This implies that only the absorptive part of the propagator is taken into account. This gives the required absorptive parts in the amplitudes. The contributions are classified according to the parton momenta. The hard-pole contribution is from figure 3 where the gluon parton carries nonzero momentum. The soft-gluon contribution is from figure 4 where the gluon parton carries zero momentum. The soft-quark contribution is from figure 5 where the quark parton is with zero momentum.

All contributions from these diagrams involve only the twist-2 gluon fragmentation function. The general structure of each type of the contributions from these diagrams can be written in the form

$$
\left.\frac{1}{m_{A}} F_{q T}\right|_{L}=\int \frac{d z}{z^{2}} d_{g}(z) d^{4} k_{A} d^{4} k_{1} \mathcal{H}_{L, k l}^{a, \rho}\left(k_{A}, k_{1}\right) \mathcal{M}_{\rho, l k}^{a}\left(k_{A}, k_{1}\right),
$$

with the quark-gluon correlator:

$$
\mathcal{M}^{a, \rho}\left(k_{A}, k_{1}\right)=g_{s} \int \frac{d^{4} \xi_{1} d^{4} \xi_{2}}{(2 \pi)^{8}} e^{-i \xi_{1} \cdot k_{A}+i \xi_{2} \cdot k_{1}}\left\langle h_{A}\left|\bar{q}(0) G^{a, \rho}\left(\xi_{2}\right) q\left(\xi_{1}\right)\right| h_{A}\right\rangle .
$$



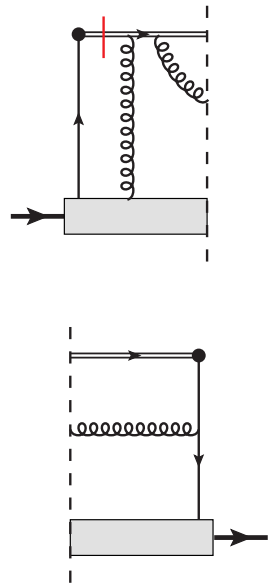
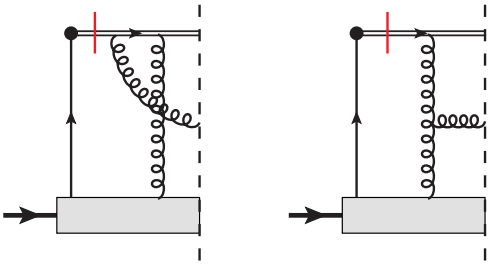

$(a)$

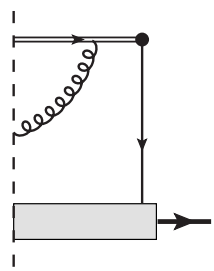

(b)

Figure 3. Diagrams for hard-pole contribution. (a) The left part of diagrams, where there is one gluon attached to the lower bubble. (b) The right-part of diagrams. The contribution is given by the interference between the left- and right diagrams.

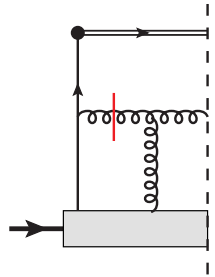

(a)

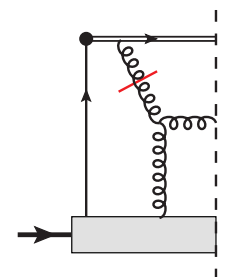

(b)

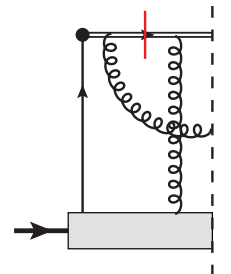

(c)

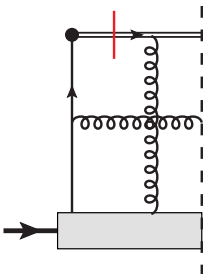

$(d)$

Figure 4. The left parts of diagrams for soft-gluon-pole contribution.

$d_{g}$ is the gluon fragmentation function. $\mathcal{H}_{L}^{a, \rho}$ is the sum of upper parts of all diagrams in which the gluon line from the correlator in the left side of the cut, or in the left part of diagrams. The quark in the right part of the diagrams is with the momentum $k_{A}$, the gluon is with $k_{1}$ and the quark in the left part is with the momentum $k_{A}-k_{1}$.

The quark-gluon density matrix can be written in the form:

$$
\begin{aligned}
\mathcal{M}^{a, \rho}\left(k_{A}, k_{1}\right)=\frac{1}{N_{c}^{2}-1} T^{a}[ & \left(M^{\rho}\left(k_{A}, k_{1}\right) \gamma^{-}+M_{A}^{\rho}\left(k_{A}, k_{1}\right) i \gamma_{5} \gamma^{-}\right) \\
& \left.+\left(M_{\perp}^{\rho \mu}\left(k_{A}, k_{1}\right) \gamma_{\perp \mu}+M_{A \perp}^{\rho \mu}\left(k_{A}, k_{1}\right) i \gamma_{5} \gamma_{\perp \mu}\right)\right]+\cdots,
\end{aligned}
$$

where $\cdots$ denote the terms beyond twist- 3 . The quark-gluon matrix elements are

$$
\begin{aligned}
M^{\rho}\left(k_{A}, k_{1}\right) & =g_{s} \int \frac{d^{4} \xi_{1} d^{4} \xi_{2}}{2(2 \pi)^{8}} e^{-i \xi_{1} \cdot k_{A}+i \xi_{2} \cdot k_{1}}\left\langle h_{A}\left|\bar{q}\left(\xi_{1}\right) \gamma^{+} G^{\rho}\left(\xi_{2}\right) q(0)\right| h_{A}\right\rangle, \\
M_{A}^{\rho}\left(k_{A}, k_{1}\right) & =-i g_{s} \int \frac{d^{4} \xi_{1} d^{4} \xi_{2}}{2(2 \pi)^{8}} e^{-i \xi_{1} \cdot k_{A}+i \xi_{2} \cdot k_{1}}\left\langle h_{A}\left|\bar{q}\left(\xi_{1}\right) \gamma^{+} \gamma_{5} G^{\rho}\left(\xi_{2}\right) q(0)\right| h_{A}\right\rangle, \\
M_{\perp}^{\rho \mu}\left(k_{A}, k_{1}\right) & =g_{s} \int \frac{d^{4} \xi_{1} d^{4} \xi_{2}}{2(2 \pi)^{8}} e^{-i \xi_{1} \cdot k_{A}+i \xi_{2} \cdot k_{1}}\left\langle h_{A}\left|\bar{q}\left(\xi_{1}\right) \gamma_{\perp}^{\mu} G^{\rho}\left(\xi_{2}\right) q(0)\right| h_{A}\right\rangle, \\
M_{A \perp}^{\rho \mu}\left(k_{A}, k_{1}\right) & =-i g_{s} \int \frac{d^{4} \xi_{1} d^{4} \xi_{2}}{2(2 \pi)^{8}} e^{-i \xi_{1} \cdot k_{A}+i \xi_{2} \cdot k_{1}}\left\langle h_{A}\left|\bar{q}\left(\xi_{1}\right) \gamma_{\perp}^{\mu} \gamma_{5} G^{\rho}\left(\xi_{2}\right) q(0)\right| h_{A}\right\rangle .
\end{aligned}
$$


The power counting for the parton momenta and the quark-gluon matrix elements are:

$$
\begin{array}{rlrl}
k_{A}^{\mu} & \sim\left(1, \lambda^{2}, \lambda, \lambda\right), & k_{1}^{\mu} & \sim\left(1, \lambda^{2}, \lambda, \lambda\right), \\
M_{\perp}^{\rho \mu} & \sim M_{A \perp}^{\rho \mu} \sim \lambda\left(1, \lambda^{2}, \lambda, \lambda\right), \quad M^{\rho} & \sim M_{A}^{\rho} \sim\left(1, \lambda^{2}, \lambda, \lambda\right) .
\end{array}
$$

In the case of $F_{q T}$, the matrix elements $M_{\perp}^{\rho \mu}$ and $M_{A \perp}^{\rho \mu}$ contribute at twist-3 only with $\rho=+$, where one can neglect all transverse- and --components of parton momenta. For $\rho \neq+$, the contributions from these two matrix elements are beyond twist-3.

We take the contributions from $M^{\rho}\left(k_{A}, k_{1}\right)$ in eq. (3.4) to discuss the collinear expansion and the gauge invariance of the results. The discussion in the case of $M_{A}^{\rho}\left(k_{A}, k_{1}\right)$ is similar. To obtain twist-3 contributions we need to expand the upper parts around the parton momenta:

$$
\hat{k}_{A}^{\mu}=\left(k_{A}^{+}, 0,0,0\right), \quad \hat{k}_{1}^{\mu}=\left(k_{1}^{+}, 0,0,0\right) .
$$

The upper part of the contributions involving $M^{\rho}\left(k_{A}, k\right)$ is given by:

$$
H_{L}^{\mu}\left(k_{A}, k_{1}\right)=\frac{1}{\left(N_{c}^{2}-1\right)} \operatorname{Tr}\left[T^{a} \gamma^{-} \mathcal{H}_{L}^{a, \mu}\left(k_{A}, k_{1}\right)\right] .
$$

The collinear expansion is:

$$
H_{L}^{\mu}\left(k_{A}, k_{1}\right)=H_{L}^{\mu}\left(\hat{k}_{A}, \hat{k}_{1}\right)+k_{1 \perp}^{\alpha} \frac{\partial H_{L}^{\mu}}{\partial k_{1 \perp}^{\alpha}}\left(\hat{k}_{A}, \hat{k}_{1}\right)+k_{A \perp}^{\alpha} \frac{\partial H_{L}^{\mu}}{\partial k_{A \perp}^{\alpha}}\left(\hat{k}_{A}, \hat{k}_{1}\right)+\cdots
$$

where terms represented by $\cdots$ give the contributions beyond twist-3. With the expansion, the contribution at the leading order of $\lambda$ can be written in the form:

$$
\begin{aligned}
\left.\frac{1}{m_{A}} F_{q T}\right|_{L}= & g_{s} \int d k_{A}^{+} d k_{1}^{+} \frac{d z}{z^{2}} d_{g}(z) \int \frac{d \lambda_{1} d \lambda_{2}}{2(2 \pi)^{2}} e^{-i \lambda_{1} k_{A}^{+}+i \lambda_{2} k_{1}^{+}}\{ \\
& i \frac{\partial H_{L}^{-}}{\partial k_{1 \perp}^{\alpha}}\left(\hat{k}_{A}, \hat{k}_{1}\right)\left\langle h_{A}\left|\bar{q}\left(\lambda_{1}\right) \gamma^{+}\left[\partial_{\perp}^{\alpha} G^{+}-\partial^{+} G_{\perp}^{\alpha}\right]\left(\lambda_{2} n\right) q(0)\right| h_{A}\right\rangle \\
& -i \frac{\partial H_{L}^{-}}{\partial k_{A \perp}^{\alpha}}\left(\hat{k}_{A}, \hat{k}_{1}\right)\left\langle h_{A}\left|\left(\partial_{\perp}^{\alpha} \bar{q}\right)\left(\lambda_{1}\right) \gamma^{+} G^{+}\left(\lambda_{2} n\right) q(0)\right| h_{A}\right\rangle \\
& \left.+\left[k_{1}^{+} \frac{\partial H_{L}^{-}}{\partial k_{1 \perp}^{\alpha}}\left(\hat{k}_{A}, \hat{k}_{1}\right)+H_{L \perp \alpha}\left(\hat{k}_{A}, \hat{k}_{1}\right)\right]\left\langle h_{A}\left|\bar{q}\left(\lambda_{1}\right) \gamma^{+} G_{\perp}^{\alpha}\left(\lambda_{2} n\right) q(0)\right| h_{A}\right\rangle\right\},
\end{aligned}
$$

where contributions beyond the leading power of $\lambda$ are neglected.

It is clear that the first term in eq. (3.9) is gauge-invariant at the considered order of $g_{s}$. The second- and third terms in eq. (3.9) are not gauge-invariant. However, because of the additional cut, i.e., that the propagator with a short bar represents an on-shell particle, one can find the following Ward identity and the identity with the momenta $\hat{k}_{1, A}$ :

$$
k_{1 \rho} H_{L}^{\rho}\left(k_{A}, k_{1}\right)=0, \quad k_{1}^{+} H_{L}^{-}\left(\hat{k}_{A}, \hat{k}_{1}\right)=0 .
$$

With the first identity, one can show that in the hard-pole- and soft-quark contributions the second- and third gauge-variant terms are zero. In the case of the soft-gluon pole contribution, because of that $k_{1}^{+}=0$ one can not use the argument of the identity. But, we find by adding the contribution from complex conjugated diagrams that the final result is gauge invariant. The situation here is similar to the analysis of twist-3 contribution of SIDIS in [36]. For the contributions from $M_{A}^{\rho}\left(k_{A}, k_{1}\right)$ the results are similar except the soft-gluon-pole contribution which is nonzero and gauge-variant. 


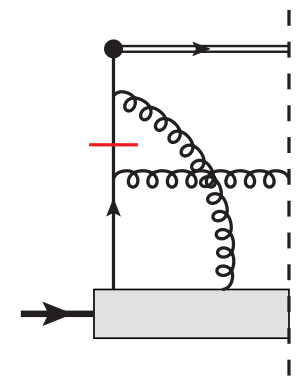

$(a)$

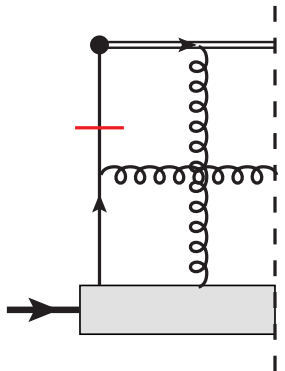

$(b)$

Figure 5. The left parts of diagrams for soft-fermion-pole contribution.

For the contributions from the matrix elements $M_{\perp}^{\rho \mu}$ and $M_{A \perp}^{\rho \mu}$ with $\rho=+$ there are similar identities to those in eq. (3.10). In the hard-pole- and soft-quark contributions only the momentum component $k_{1}^{+}$and $k_{A}^{+}$are not zero. Therefore, there are no hard-pole- and soft-quark contributions from these two matrix elements because of an identity similar to the second one in eq. (3.10). For soft-gluon-contributions, the argument can not be used because of that $k_{1}^{+}=0$. With explicit calculations we find that the soft-gluon-pole contribution from $M_{\perp}^{+\mu}$ is zero if we add the contribution from complex conjugated diagrams. The soft-gluon-pole contribution from $M_{A \perp}^{+\mu}$ and that from $M_{A}^{\rho}$ are not zero. Both contributions can not be written in a gauge-invariant form. We notice that in the matrix element $M_{A \perp}^{+\mu}$ one of the quark fields must be the --component with the decomposition discussed in the next section. With equation of motion one can show that the sum of soft-gluon-pole contributions from $M_{A \perp}^{+\mu}$ and $M_{A}^{\rho}$ can be written in a gauge invariant form, i.e., in terms of $T_{F}$. Therefore, the soft-gluon-pole contributions come from all matrix elements in eq. (3.4) except $M_{\perp}^{\rho \mu}$ with $\rho=+$. The calculation is tedious but straightforward. Hence, we will give our results of this section directly without giving the details of our calculations.

The hard-pole contribution is from figure 3. The result is:

$$
\left.\frac{1}{m_{A}} F_{q T}\left(x, \xi, k_{\perp}\right)\right|_{H P}=g_{s}^{2} \frac{N_{c}}{\left(k_{\perp}^{2}\right)^{2}} \int \frac{d z}{z^{2}} d_{g}(z) \frac{z^{2}}{y}\left(\xi T_{\Delta}(y, x)-(\xi+2 x z) T_{F}(y, x)\right) .
$$

The soft-gluon-pole contribution is from figure 4 . The result is

$$
\begin{array}{r}
\left.\frac{1}{m_{A}} F_{q T}\left(x, \xi, k_{\perp}\right)\right|_{S G P}=\frac{g_{s}^{2} N_{c}}{\left(k_{\perp}^{2}\right)^{2}} \int \frac{d z}{z^{2}} d_{g}(z) \frac{1}{y^{3}}\left[z^{3}\left(y^{3}+3 x^{2} y-2 x^{3}\right) T_{F}(y, y)\right. \\
\left.-y \xi z^{2}\left(y^{2}+x^{2}\right) \frac{\partial T_{F}(y, y)}{\partial y}\right] .
\end{array}
$$

There is no soft-gluon contribution from $T_{\Delta}$ because of that $T_{\Delta}(y, y)=0$. The soft-quark contribution is from figure 5 . The result is

$$
\left.\frac{1}{m_{A}} F_{q T}\left(x, \xi, k_{\perp}\right)\right|_{S F P}=g_{s}^{2} \frac{1}{N_{c}} \frac{1}{\left(k_{\perp}^{2}\right)^{2}} \int \frac{d z}{z^{2}} d_{g}(z) \frac{x \xi z}{y^{3}}\left((x z-\xi) T_{F}(y, 0)-(\xi+x z) T_{\Delta}(y, 0)\right) .
$$

In these results $y$ is given by $y=x+\xi / z$. 

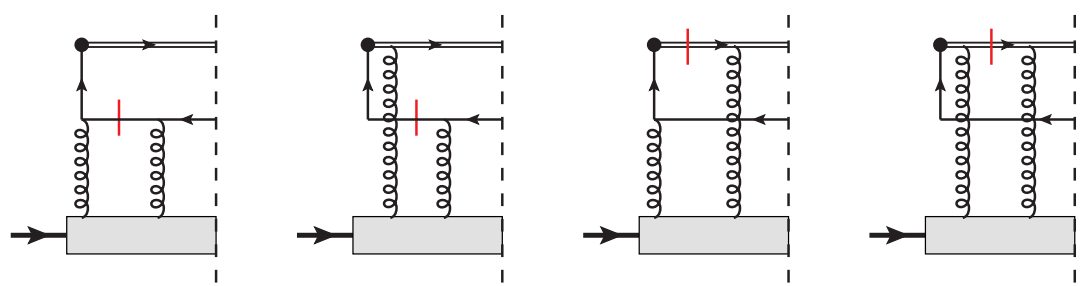

(a)
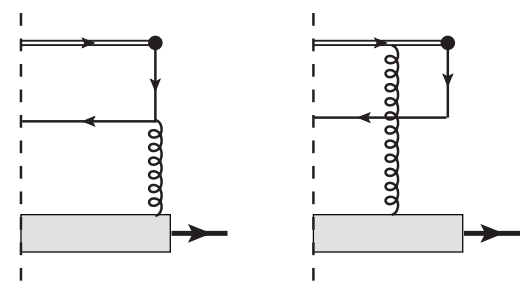

(b)

Figure 6. Diagrams for twist-3 gluonic contribution.

Besides the twist-3 contributions from quark-gluon correlators, there are contributions from purely gluonic distributions at twist-3. Since an additional cut or an absorptive part is required, there is no contribution from figure $2 \mathrm{~b}$. The contributions are only from figure 6 , where one quark propagator or eikonal propagator is with a short bar.

After completing the collinear expansion related to the produced hadron, the contribution from figure 6 can be written in the form:

$$
\begin{aligned}
\left.\frac{1}{m_{A}} F_{q T}\left(x, \xi, k_{\perp}\right)\right|_{L}= & \int \frac{d z}{z^{2}} d_{\bar{q}}(z) d^{4} k_{1} d^{4} k_{2} H_{L \mu_{1} \mu_{2} \mu_{3}}^{a b c}\left(k_{1}, k_{2}\right) \\
& \int \frac{d^{4} x_{1} d^{4} x_{2}}{(2 \pi)^{8}} e^{i x_{1} \cdot k_{1}+i x_{2} \cdot k_{2}}\left\langle h_{A}\left|G^{c, \mu_{3}}(0) G^{b, \mu_{2}}\left(x_{2}\right) G^{a, \mu_{1}}\left(x_{1}\right)\right| h_{A}\right\rangle .
\end{aligned}
$$

This contribution is associated with the fragmentation function of an antiquark into the observed hadron. $H_{L \mu_{1} \mu_{2} \mu_{3}}^{a b c}\left(k_{1}, k_{2}\right)$ is the perturbative part which is the sum of the upper parts of figure 6 . In figure 6 a the left gluon line carries the momentum $k_{1}$ and the right one carries $k_{2}$. In figure $6 \mathrm{~b}$, the gluon line carries the momentum $k_{3}$. Because of the cut and that the propagator with a short bar represents an on-shell particle, one can find the following identities:

$$
k_{1}^{\mu_{1}} H_{L \mu_{1} \mu_{2} \mu_{3}}^{a b c}\left(k_{1}, k_{2}\right)=0, \quad k_{3}^{\mu_{3}} H_{L \mu_{1} \mu_{2} \mu_{3}}^{a b c}\left(k_{1}, k_{2}\right)=0, \quad k_{3}=-\left(k_{1}+k_{2}\right) .
$$

The contributions from the last two diagrams in figure 6 a are proportional to $\delta\left(k_{2}^{+}\right)$because of the cut on the eikonal propagator. With this fact we can find the identity:

$$
k_{2}^{\mu_{2}} H_{L \mu_{1} \mu_{2} \mu_{3}}^{a b c}\left(k_{1}, k_{2}\right)=0 .
$$

However, this identity is useless in the case of soft-gluon-pole contributions here. This is similar to the case discussed after eq. (3.10). We find the leading contribution from figure 6 and their complex conjugated diagrams can be written in a gauge-invariant form. Other 
contributions are beyond twist-3. We have the result with the twist-3 gluon distributions:

$$
\begin{aligned}
\frac{1}{m_{A}} & \left.F_{q T}\left(x, \xi, k_{\perp}\right)\right|_{3 G} \\
= & \frac{4 \pi g_{s}^{2} \xi^{2}}{\left(k_{\perp}^{2}\right)^{2}} \int \frac{d z}{z^{2}} d_{\bar{q}}(z) \frac{1}{z y^{5}}\left[2\left(\xi^{2}+2 x^{2} z^{2}-\xi x z\right)(N(y, y)-O(y, y))\right. \\
& -2\left(\xi^{2}+4 x^{2} z^{2}-3 \xi x z\right)(N(y, 0)+O(y, 0))+y(\xi-x z)^{2} \frac{d}{d y}(N(y, 0)+O(y, 0)) \\
& \left.-y\left(\xi^{2}+x^{2} z^{2}\right) \frac{d}{d y}(N(y, y)-O(y, y))\right]
\end{aligned}
$$

with $y=x+\xi / z$.

The final result for $F_{q T}$ is the sum:

$$
\frac{1}{m_{A}} F_{q T}\left(x, \xi, k_{\perp}\right)=\frac{1}{m_{A}}\left(\left.F_{q T}\right|_{H P}+\left.F_{q T}\right|_{S G P}+\left.F_{q T}\right|_{S F P}+\left.F_{q T}\right|_{3 G}\right)\left(x, \xi, k_{\perp}\right),
$$

where terms in the sum can be found in eqs. (3.11), (3.12), (3.13), (3.17).

\section{Matching of $\Delta F_{q T}$}

$\Delta F_{q T}$ represents the double spin asymmetry. Unlike $F_{q T}$, the asymmetry is not zero in the absence of absorptive parts in the scattering amplitude. In the matching of $\Delta F_{q T}$, there are contributions from figure 2 . We first discuss the contribution from figure $2 \mathrm{a}$. It involves only gluon fragmentation function and can be written in the form:

Fig. $2 a=\int d^{4} p \frac{d z}{z^{2}} \delta\left(x P^{+}-p^{+}+k_{g}^{+}\right) d_{g}(z) H_{k l}(p) \int \frac{d^{4} \xi}{(2 \pi)^{4}} e^{-i \xi \cdot p}\left\langle h_{A}\left|\bar{q}_{k}(\xi) q_{l}(0)\right| h_{A}\right\rangle$,

where $k_{g}$ is the momentum of the gluon which is fixed as $z k_{g}=k . p$ is the momentum of the right quark line. $H_{k l}(p)$ is the sum of the perturbative parts represented by the upper parts of the diagrams in figure 2a. With the power counting in eq. (3.5) for parton momenta we can expand $H_{k l}(p)$ around the momentum $\hat{p}^{\mu}=\left(p^{+}, 0,0,0\right)$ :

$$
H_{k l}(p)=H_{k l}(\hat{p})+p_{\perp}^{\alpha} \frac{\partial H_{k l}}{\partial p_{\perp}^{\alpha}}(\hat{p})+\cdots,
$$

where $\cdots$ stand for contributions at higher orders. Taking the leading term and the twist-2 part of the quark density matrix in eq. (2.14), one obtains the twist-2 contribution to $F_{q}$ and $\Delta F_{q}$ given as the first terms in eq. (2.16).

The twist- 3 contribution is obtained by taking the second term in the expansion in eq. (4.2) or the first term combined with the twist-3 part of the quark density matrix. The result is:

$$
\begin{aligned}
& \left.\frac{2 k_{\perp} \cdot s_{\perp}}{m_{A}} \Delta F_{q T}\left(x, \xi, k_{\perp}\right)\right|_{2 a} \\
& =\frac{k_{\perp \mu}}{\left(k_{\perp}^{2}\right)^{2}} \int \frac{d z}{z^{2}} d_{g}(z)\left[H_{2 p, T}(x, \xi) \int \frac{d \lambda}{4 \pi} P^{+} e^{-i y P^{+}}\left\langle h_{A}\left|\bar{q}(\lambda n) \gamma_{\perp}^{\mu} \gamma_{5} q(0)\right| h_{A}\right\rangle\right. \\
& \left.\quad+H_{2 p, \partial}(x, \xi) \int \frac{d \lambda}{4 \pi} i e^{-i y P^{+}}\left\langle h_{A}\left|\bar{q}(\lambda n) \gamma^{+} \gamma_{5} \partial_{\perp}^{\mu} q(0)\right| h_{A}\right\rangle\right]
\end{aligned}
$$




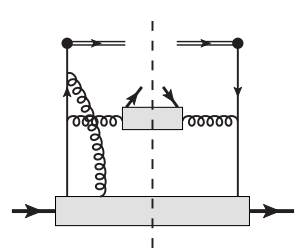

$\left(a_{1}\right)$

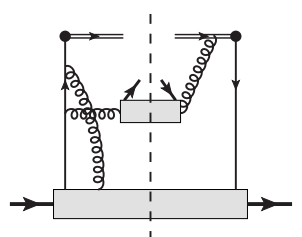

$\left(a_{2}\right)$

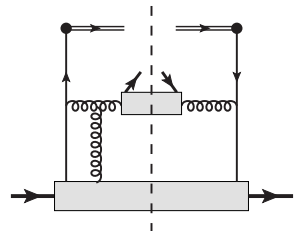

$\left(b_{1}\right)$

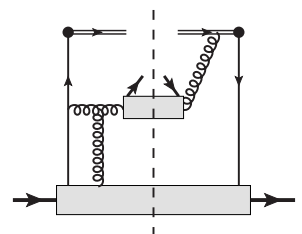

$\left(b_{2}\right)$

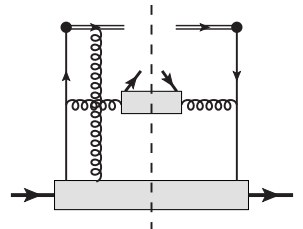

$\left(c_{1}\right)$

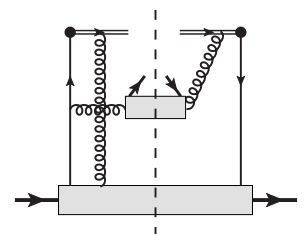

$\left(c_{2}\right)$

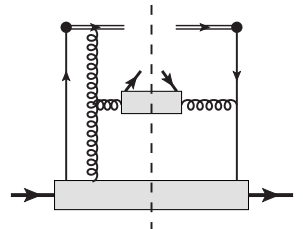

$\left(d_{1}\right)$

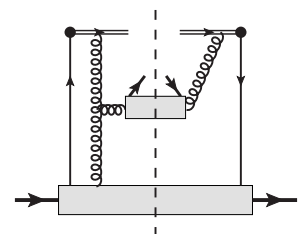

$\left(d_{2}\right)$

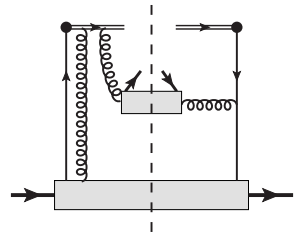

$\left(e_{1}\right)$

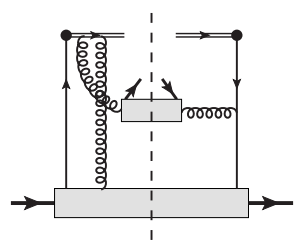

$\left(e_{2}\right)$

Figure 7. Diagrams for the matching of $\Delta F_{q T}$.

with

$$
H_{2 p, \partial}(x, \xi)=-8 g_{s}^{2} C_{F} \frac{\xi z^{2}\left(y^{2}+2 x^{2}\right)}{y^{3}}, \quad H_{2 p, T}(x, \xi)=8 g_{s}^{2} C_{F} \frac{\xi x^{2} z^{2}}{y^{2}}, \quad y=x+\xi / z .
$$

These contributions seems to be with the twist-3 distributions $q_{T}$ and $q_{\partial}$, respectively. But, they are not exactly those distributions. The contributions of gauge links are not included. When we consider the contributions in figure 7 where an additional gluon exchanges, parts of them will be the contributions of gauge links at the considered order.

In calculating the contributions with one-gluon exchange given by figure 7 , we have the same quark-gluon correlator given in eq. (3.2) and its decomposition in eq. (3.4). By calculating the contribution with $M_{A}^{\rho}$, we find that a part of the contribution can be added to the second term in eq. (4.3) so that the sum is obtained by inserting gauge links in the matrix element at the considered order of $g_{s}$. With the gauge links the matrix element is that used to defined $q_{\partial}$. Excluding this part, the contribution from figure 7 with $M_{A}^{\rho}$ is:

$$
\begin{aligned}
& \left.\frac{2 k_{\perp} \cdot s_{\perp}}{m_{A}} \Delta F_{q T}\left(x, \xi, k_{\perp}\right)\right|_{M_{A}^{\rho}} \\
& =\left(-i g_{s}\right) \int d k_{A}^{+} d k_{1}^{+} \frac{d z}{z^{2}} d_{g}(z) \int \frac{d \lambda_{1} d \lambda_{2}}{2(2 \pi)^{2}} e^{-i \lambda_{1} k_{A}^{+}+i \lambda_{2} k_{1}^{+}}\left\{\frac{i}{k_{1}^{+}} H_{\perp A L \alpha}\left(\hat{k}_{A}, \hat{k}_{1}\right)\right. \\
& \quad\left\langle h_{A}\left|\bar{q}\left(\lambda_{1} n\right) \gamma^{+} \gamma_{5}\left[\partial^{+} G_{\perp}^{\alpha}-\partial_{\perp}^{\alpha} G^{+}\right]\left(\lambda_{2} n\right) q(0)\right| h_{A}\right\rangle+h . c . \\
& \quad+F_{0}(x, \xi) \frac{1}{k_{1}^{+}} k_{\perp}^{\alpha}\left[\delta\left(k_{A}^{+}-k_{0}^{+}\right)\left\langle h_{A}\left|\bar{q}\left(\lambda_{1} n\right) \gamma^{+} \gamma_{5} G^{+}\left(\lambda_{2} n\right) \partial_{\perp \alpha} q(0)\right| h_{A}\right\rangle\right. \\
& \left.\left.\quad+\delta\left(k_{A}^{+}-k_{1}^{+}-k_{0}^{+}\right)\left\langle h_{A}\left|\partial_{\perp \alpha} \bar{q}\left(\lambda_{1} n\right) \gamma^{+} \gamma_{5} G^{+}\left(\lambda_{2} n\right) q(0)\right| h_{A}\right\rangle\right]\right\}
\end{aligned}
$$

with

$$
\tilde{H}_{0}^{\alpha}(x, \xi)=\tilde{k}_{\perp}^{\alpha} F_{0}(x, \xi), \quad H_{0}^{\alpha}(x, \xi)=k_{\perp}^{\alpha} F_{0}(x, \xi), \quad F_{0}(x, \xi)=g_{s}^{2} C_{A} \frac{\xi z^{3}(\xi+2 x z)}{\left(k_{\perp}^{2}\right)^{2}(\xi+x z)^{2}} .
$$


In eq. (4.5), the first two terms are gauge invariant, while the third- and fourth terms are not. The contribution from $M^{\rho}$ in the quark-gluon density matrix can be written:

$$
\begin{aligned}
& \left.\frac{2 k_{\perp} \cdot s_{\perp}}{m_{A}} \Delta F_{q T}\left(x, \xi, k_{\perp}\right)\right|_{M^{\rho}} \\
& =g_{s} \int d k_{A}^{+} d k_{1}^{+} \frac{d z}{z^{2}} d_{g}(z) \int \frac{d \lambda_{1} d \lambda_{2}}{2(2 \pi)^{2}} e^{-i \lambda_{1} k_{A}^{+}+i \lambda_{2} k_{1}^{+}}\left\{\frac{i}{k_{1}^{+}} H_{\perp L \alpha}\left(\hat{k}_{A}, \hat{k}_{1}\right)\right. \\
& \quad\left\langle h_{A}\left|\bar{q}\left(\lambda_{1} n\right) \gamma^{+}\left[\partial^{+} G_{\perp}^{\alpha}-\partial_{\perp}^{\alpha} G^{+}\right]\left(\lambda_{2} n\right) q(0)\right| h_{A}\right\rangle+h . c . \\
& \quad+F_{0}(x, \xi) \frac{1}{k_{1}^{+}} \tilde{k}_{\perp}^{\alpha}\left[\delta\left(k_{A}^{+}-k_{0}^{+}\right)\left\langle h_{A}\left|\bar{q}\left(\lambda_{1} n\right) \gamma^{+} G^{+}\left(\lambda_{2} n\right) \partial_{\perp \alpha} q(0)\right| h_{A}\right\rangle\right. \\
& \left.\left.\quad+\delta\left(k_{A}^{+}-k_{1}^{+}-k_{0}^{+}\right)\left\langle h_{A}\left|\partial_{\perp \alpha} \bar{q}\left(\lambda_{1} n\right) \gamma^{+} G^{+}\left(\lambda_{2} n\right) q(0)\right| h_{A}\right\rangle\right]\right\},
\end{aligned}
$$

where the first two terms are gauge invariant. The third- and fourth terms are not gauge invariant. It is interesting to note that they have the same perturbative coefficient function $F_{0}$ as that in the gauge variant contribution in eq. (4.5).

Now we consider the contributions with $M_{\perp}^{\rho \mu}$ and $M_{A \perp}^{\rho \mu}$ in the quark-gluon density matrix. The twist-3 contributions are given by taking the index $\rho=+$, i.e, only the field component $G^{+}$is involved. We find that the contribution from $M_{\perp}^{\rho \mu}$ is proportional to $C_{A}$. In the contribution from $M_{A \perp}^{\rho \mu}$, there is a part proportional to $C_{F}$. This part can be summed with the first term in eq. (4.3). The sum is obtained by inserting gauge links in the matrix element in the first term so that the matrix element is that used to defined $q_{T}$. The sum is then gauge invariant at the considered order of $g_{s}$. The sum of the remaining contribution from $M_{A \perp}^{\rho \mu}$ and that from $M_{\perp}^{\rho \mu}$ can be written in the form:

$$
\begin{aligned}
\left.\frac{2 k_{\perp} \cdot s_{\perp}}{m_{A}} \Delta F_{q T}\left(x, \xi, k_{\perp}\right)\right|_{C_{A}}= & g_{s} \int \frac{d z}{z^{2}} d_{g}(z) F_{0}(x, \xi) \int d k_{A}^{+} d k_{1}^{+}\left(-1+\frac{k_{0}^{+}}{k_{1}^{+}}\right) \delta\left(k_{A}^{+}-k_{0}^{+}\right) \\
& \int \frac{d \lambda_{1} d \lambda_{2}}{2(2 \pi)^{2}} e^{-i \lambda_{1} k_{A}^{+}+i \lambda_{2} k_{1}^{+}}\left\langle h_{A}\right| \bar{q}\left(\lambda_{1} n\right)\left(\gamma \cdot k_{\perp} \gamma_{5}\right. \\
& \left.\left.\left.+i \gamma \cdot \tilde{k}_{\perp}\right) G^{+}\left(\lambda_{2} n\right) q(0) \mid h_{A}\right)\right\rangle+ \text { h.c. }
\end{aligned}
$$

This sum is not gauge invariant and it involves the same perturbative function $F_{0}$. We notice that the quark field can be written as the sum of a +- and --component:

$$
q^{(+)}(x)=\frac{1}{2} \gamma^{-} \gamma^{+} q(x), \quad q^{(-)}(x)=\frac{1}{2} \gamma^{+} \gamma^{-} q(x), \quad q(x)=q^{(+)}(x)+q^{(-)}(x) .
$$

The --component is not independent. With the equation of motion one has:

$$
q^{(-)}(x)=\frac{1}{2} \mathcal{L}_{n}^{\dagger}(x) \int_{0}^{\infty} d \lambda\left(\mathcal{L}_{n} \gamma^{+} \gamma_{\perp}^{\mu} D_{\mu} q^{(+)}\right)(\lambda n+x) .
$$

In eq. (4.8) one quark field has to be the --component. Using the solution in eq. (4.10) one 
has the sum:

$$
\begin{aligned}
\left.\frac{2 k_{\perp} \cdot s_{\perp}}{m_{A}} \Delta F_{q T}\left(x, \xi, k_{\perp}\right)\right|_{C_{A}}= & g_{s} \int \frac{d z}{z^{2}} d_{g}(z) F_{0}(x, \xi) \int d k_{A}^{+} d k_{1}^{+} \frac{i}{k_{1}^{+}} \int \frac{d \lambda_{1} d \lambda_{2}}{2(2 \pi)^{2}} e^{-i \lambda_{1} k_{A}^{+}+i \lambda_{2} k_{1}^{+}} \\
& {\left[\delta\left(k_{A}^{+}-k_{0}^{+}\right)\left\langle h_{A}\left|\bar{q}\left(\lambda_{1} n\right)\left(k_{\perp}^{\alpha} \gamma^{+} \gamma_{5}+i \tilde{k}_{\perp}^{\alpha} \gamma^{+}\right) G^{+}\left(\lambda_{2} n\right) \partial_{\perp \alpha} q(0)\right| h_{A}\right\rangle\right.} \\
& +\delta\left(k_{A}^{+}-k_{1}^{+}-k_{0}^{+}\right)\left\langle h_{A}\right| \partial_{\perp \alpha} \bar{q}\left(\lambda_{1} n\right)\left(k_{\perp}^{\alpha} \gamma^{+} \gamma_{5}\right. \\
& \left.\left.-i \tilde{k}_{\perp}^{\alpha} \gamma^{+}\right) G^{+}\left(\lambda_{2} n\right) q(0)\left|h_{A}\right\rangle\right] .
\end{aligned}
$$

Comparing the sum with the gauge variant contribution in eq. (4.5), (4.7), we find that all gauge variant contributions are cancelled each other so that the remaining contribution is gauge invariant. We have then the result which is gauge invariant:

$$
\begin{aligned}
\left.\frac{1}{m_{A}} \Delta F_{q T}\left(x, \xi, k_{\perp}\right)\right|_{q \bar{q}+q G \bar{q}}= & \frac{1}{2\left(k_{\perp}^{2}\right)^{2}} \int \frac{d z}{z^{2}} d_{g}(z)\left[\left(H_{2 p, T}(x, \xi) q_{T}(y)+H_{2 p, \partial}(x, \xi) q_{\partial}(y)\right)\right. \\
& \left.+\frac{2}{\pi} \int d x_{2}\left(T_{F}\left(y, x_{2}\right) H\left(x, \xi, x_{2}\right)+T_{\Delta}\left(y, x_{2}\right) H_{A}\left(x, \xi, x_{2}\right)\right)\right]
\end{aligned}
$$

with $H_{2 p, \partial}$ and $H_{2 p, T}$ are given in eq. (4.3). $H\left(x, \xi, x_{2}\right)$ and $H_{A}\left(x, \xi, x_{2}\right)$ are given as:

$$
\begin{aligned}
H\left(x, \xi, x_{2}\right) & =\frac{2 g_{s}^{2} \xi z^{2}}{y x_{2}\left(y-x_{2}\right)}\left[C_{A} \frac{x^{2}-x_{2} y}{x_{2}-x}+2 C_{F} \frac{1}{y}\left(x y-x_{2}(x+y)\right)\right] \\
H_{A}\left(x, \xi, x_{2}\right) & =\frac{2 g_{s}^{2} \xi z^{2}}{y x_{2}\left(x_{2}-y\right)}\left[C_{A} \frac{\left(x^{2}+x_{2} y\right)\left(x_{2}+y-2 x\right)}{\left(x_{2}-x\right)\left(x_{2}-y\right)}+2 C_{F} \frac{1}{y}\left(x(2 x-y)+x_{2}(x+y)\right)\right] .
\end{aligned}
$$

$\Delta F_{q T}$ receives contributions from twist-3 gluon distributions, where the antiquark fragmentation function is involved. These contributions are given by diagrams of two-gluon exchanges as given in figure $2 \mathrm{~b}$ and by three-gluon exchanges in figure 6 without the cut on the quark propagator and the gauge link. It is noted that the contribution of threegluon exchanges from the second- and third diagram in figure $6 \mathrm{a}$ is the same. Because of Bose-symmetry, one should either take any one of the two diagrams, or the half of the sum into account.

The contribution of two-gluon exchanges from figure $2 \mathrm{~b}$ can be written in the form:

$$
\text { Fig. } 2 \mathrm{~b}=\int \frac{d z}{z^{2}} d_{\bar{q}}(z) d^{4} k_{1} H^{\mu \nu}\left(k_{1}\right) \int \frac{d^{4} x}{(2 \pi)^{4}} e^{i x \cdot k_{1}}\left\langle h_{A}\left|G_{\mu}^{a}(0) G_{\nu}^{a}(x)\right| h_{A}\right\rangle,
$$

where $H^{\mu \nu}\left(k_{1}\right)$ is the sum of the perturbative parts represented by the upper parts of the diagrams in figure $2 \mathrm{~b} . k_{1}$ is the momentum carried by the gluons. It is easy to check the following Ward identities:

$$
k_{1 \mu} H^{\mu \nu}\left(k_{1}\right)=0, \quad k_{1 \nu} H^{\mu \nu}\left(k_{1}\right)=0 .
$$


Before doing the collinear expansion in $k_{1}$ around $\hat{k}_{1}^{\mu}=\left(k_{1}^{+}, 0,0,0\right)$, we can use these identities to manipulate the expression:

$$
\begin{aligned}
H^{\mu \nu} & \left(k_{1}\right) \int \frac{d^{4} x}{(2 \pi)^{4}} e^{i x \cdot k_{1}}\left\langle h_{A}\left|G_{\mu}^{a}(0) G_{\nu}^{a}(x)\right| h_{A}\right\rangle \\
= & \frac{1}{\left(k_{1}^{+}\right)^{2}} \int \frac{d^{4} x}{(2 \pi)^{4}} e^{i x \cdot k_{1}}\left(H^{\mu_{\perp} \alpha_{\perp}}\left(k_{1}\right)\left\langle h_{A}\left|\hat{G}^{a,+}{ }_{\mu_{\perp}}(0) \hat{G}^{a,+}{ }_{\alpha_{\perp}}(x)\right| h_{A}\right\rangle\right. \\
& +H^{+\alpha_{\perp}}\left(k_{1}\right)\left\langle h_{A}\left|\hat{G}^{a,+-}(0) \hat{G}^{a,+}{ }_{\alpha_{\perp}}(x)\right| h_{A}\right\rangle+H^{\mu_{\perp}+}(k)\left\langle h\left|\hat{G}^{a,+}{ }_{\mu_{\perp}}(0) \hat{G}^{a,+-}(x)\right| h_{A}\right\rangle \\
& \left.+H^{++}\left(k_{1}\right)\left\langle h_{A}\left|\hat{G}^{a,+-}(0) \hat{G}^{a,+-}(x)\right| h_{A}\right\rangle\right)
\end{aligned}
$$

where $\hat{G}^{\mu \nu}=\partial^{\mu} G^{\nu}-\partial^{\nu} G^{\mu}$. In the first term, the leading contribution in the collinear expansion of $H^{\mu_{\perp} \alpha_{\perp}}\left(k_{1}\right)$ gives the twist-2 contribution, i.e., to $\Delta F_{q}$. The twist-3 contribution is obtained by expanding the next-to-leading contribution. The leading contribution of the second- and third term is at twist-3. The last term is a twist-4 contribution which can be neglected. In the collinear expansion of $H^{\mu \nu}$, we find that the next-to-leading contribution $H^{\mu_{\perp} \alpha_{\perp}}\left(k_{1}\right)$ is antisymmetric in the indices $\mu_{\perp}$ and $\alpha_{\perp}$. Because of symmetries, the related matrix element is symmetric in the indices $\mu_{\perp}$ and $\alpha_{\perp}$, as shown in eq. (2.29). Hence, the first term gives no contribution. Therefore, only the second- and third gives nonzero contribution at twist-3. The contribution can be expressed by the twist-3 gluon distribution:

$$
g_{3 T}(x) \tilde{s}^{\mu}=\frac{i}{x} \int \frac{d \lambda}{2 \pi} e^{-i x \lambda P^{+}}\left\langle h_{A}\left|G^{+-}(\lambda n) \mathcal{L}_{n}^{\dagger}(\lambda n) \mathcal{L}_{n}(0) G^{+\mu}(0)\right| h_{A}\right\rangle .
$$

We obtain the contribution from figure $2 \mathrm{~b}$ :

$$
\left.\frac{1}{m_{A}} \Delta F_{q T}\left(x, \xi, k_{\perp}\right)\right|_{2 G}=\frac{16 \pi \alpha_{s}}{\left(k_{\perp}^{2}\right)^{2}} \int \frac{d z}{z^{2}} d_{\bar{q}}(z) \frac{\xi^{2} x z}{y^{2}} g_{3 T}(y), \quad y=x+\frac{\xi}{z} .
$$

It is noted that from figure $2 \mathrm{~b}$ we only obtain the contribution with $g_{3 T}$ defined only with $\hat{G}^{\mu \nu}$ and without gauge links. The contribution alone is not gauge invariant. There is a difference of a two-gluon term between $\hat{G}^{\mu \nu}$ and $G^{\mu \nu}$. In the calculation of three-gluon exchanges given by diagrams in figure 6 , we find that a part of the contribution from figure 6 gives the needed two-gluon term in $G^{\mu \nu}$ and another part forms the gauge links in $g_{3 T}$ at the considered order of $g_{s}$.

The contribution from three-gluon exchanges is given by diagrams in figure 6 . It can be written

$$
\begin{aligned}
\text { Fig. } 6= & \int \frac{d z}{z^{2}} d_{\bar{q}}(z) \int d^{4} k_{1} d^{4} k_{2} d^{4} k_{3}^{4} H_{\mu_{1} \mu_{2} \mu_{3}}^{a b c}\left(k_{1}, k_{2}, k_{3}\right) \\
& \int \frac{d^{4} x_{1} d^{4} x_{2} d^{4} x_{3}}{(2 \pi)^{12}} e^{i k_{1} \cdot x_{1}+i k_{2} \cdot x_{2}-i k_{3} \cdot x_{3}}\left\langle h_{A}\left|G^{c, \mu_{3}}\left(x_{3}\right) G^{b, \mu_{2}}\left(x_{2}\right) G^{a, \mu_{1}}\left(x_{1}\right)\right| h_{A}\right\rangle,
\end{aligned}
$$

where $H_{\mu_{1} \mu_{2} \mu_{3}}^{a b c}\left(k_{1}, k_{2}, k_{3}\right)$ is the sum of the perturbative parts represented by the upper parts of the diagrams in figure 6 . The two gluons in figure 6a carry the momentum $k_{1}$ and 
$k_{2}$, respectively. $k_{3}$ is the momentum carried by the gluon in figure $6 \mathrm{~b}$. To obtain the gauge invariant result, we first note that there is a Ward identity related to the gluon in figure 6b:

$$
k_{3}^{\mu_{3}} H_{\mu_{1} \mu_{2} \mu_{3}}^{a b c}\left(k_{1}, k_{2}, k_{3}\right)=0 .
$$

This identity can be easily checked as those in eq. (4.15). With this identity we can write the contribution as:

$$
\begin{aligned}
\text { Fig. } 6= & \int \frac{d z}{z^{2}} d_{\bar{q}}(z) \int d^{4} k_{1} d^{4} k_{2} \frac{i}{k_{3}^{+}} H_{\mu_{1} \mu_{2} \mu_{3}}^{a b c}\left(k_{1}, k_{2}, k_{3}\right) \\
& \int \frac{d^{4} x_{1} d^{4} x_{2}}{(2 \pi)^{8}} e^{i k_{1} \cdot x_{1}+i k_{2} \cdot x_{2}}\left\langle h_{A}\left|\hat{G}^{c,+\mu_{3}}\left(x_{3}\right) G^{b, \mu_{2}}\left(x_{2}\right) G^{a, \mu_{1}}\left(x_{1}\right)\right| h_{A}\right\rangle,
\end{aligned}
$$

with $k_{3}=-k_{1}-k_{2}$. To proceed further, we notice that the perturbative coefficient function $H_{\mu_{1} \mu_{2} \mu_{3}}^{a b c}$ can be decomposed into a totally symmetric and a totally asymmetric part in the three color indices:

$$
H_{\mu_{1} \mu_{2} \mu_{3}}^{a b c}\left(k_{1}, k_{2}, k_{3}\right)=\left.H_{\mu_{1} \mu_{2} \mu_{3}}^{a b c}\left(k_{1}, k_{2}, k_{3}\right)\right|_{d}+\left.H_{\mu_{1} \mu_{2} \mu_{3}}^{a b c}\left(k_{1}, k_{2}, k_{3}\right)\right|_{f}
$$

where the first term is the symmetric part proportional to $d^{a b c}$, and the second term is antisymmetric part proportional to $f^{a b c}$. For the symmetric part, we have still the following Ward identities

$$
\left.k_{1}^{\mu_{1}} H_{\mu_{1} \mu_{2} \mu_{3}}^{a b c}\left(k_{1}, k_{2}, k_{3}\right)\right|_{d}=0,\left.\quad k_{2}^{\mu_{2}} H_{\mu_{1} \mu_{2} \mu_{3}}^{a b c}\left(k_{1}, k_{2}, k_{3}\right)\right|_{d}=0
$$

because of that the color symmetric part is essentially an amplitude of QED. With these identities one can write the contribution from the symmetric part in a gauge invariant form.

For the antisymmetric part there are no Ward identities like those in eq. (4.23). We need to do the collinear expansion first and then try to find a gauge invariant result. The calculation of this part is very tedious. One has to expand the antisymmetric part in eq. (4.22) to order of $\lambda^{2}$ in order to obtain the complete twist-3 contribution. At the end, we find that a part of the antisymmetric part gives the contributions which can be combined with the contribution of two-gluon exchange into a gauge invariant form as discussed after eq. (4.18). The remaining contribution of the antisymmetric part can be written in the form with the three-gluon correlation function $M_{F}^{\mu \nu \rho}$ defined in eq. (2.23). We have the following result from figure 6 :

$$
\begin{aligned}
\left.\frac{1}{m_{A}} \Delta F_{q T}\left(x, \xi, k_{\perp}\right)\right|_{3 G}= & \frac{g_{s}^{2}}{\left(k_{\perp}^{2}\right)^{2}} \int \frac{d z}{z^{2}} d_{\bar{q}}(z) \int d x_{2} \frac{-4 \xi^{2}}{x_{2}^{2}\left(y-x_{2}\right) y^{3}}\left\{x _ { 2 } \xi \left[O\left(y-x_{2}, y\right)\right.\right. \\
& \left.-N\left(y-x_{2}, y\right)\right]+\left(2 \xi y+y x_{2} z-y^{2} z-\xi x_{2}\right)\left[N\left(y_{2}, x_{2}\right)+O\left(y, x_{2}\right)\right] \\
& \left.+y\left(z\left(y+x_{2}\right)-2 \xi\right)\left[N\left(y-x_{2},-x_{2}\right)+O\left(y-x_{2},-x_{2}\right)\right]\right\} .
\end{aligned}
$$

The total matching result is the sum of the results in eq. (4.18), (4.24). It is noted that the twist-3 distribution $g_{3 T}$ in eq. (4.18) is not independent. Using equation of motion and the 
relation in eqs. (2.24), (2.26), $g_{3 T}$ can be expressed with the twist-3 distribution $T_{F}$ and $N$. We have:

$$
g_{3 T}(x)=-\frac{1}{x^{2}} \int d x_{2}\left\{\frac{1}{\pi} T_{F}\left(x_{2}+x, x\right)+P\left(\frac{1}{x-x_{2}}\right)\left[N\left(x, x-x_{2}\right)-N\left(x, x_{2}\right)+2 N\left(x_{2}, x_{2}-y\right)\right]\right\} .
$$

The result of $\Delta F_{q T}$ from figure $2 \mathrm{~b}$ and figure 6 is:

$$
\begin{aligned}
\frac{1}{m_{A}} \Delta & \left.F_{q T}\left(x, \xi, k_{\perp}\right)\right|_{2 G+3 G} \\
= & \frac{-16 \pi \alpha_{s} \xi^{2}}{\left(k_{\perp}^{2}\right)^{2}} \int \frac{d z d x_{2}}{z^{2} y^{3}} d_{\bar{q}}(z)\left\{\frac{x z}{\pi y} T_{F}\left(x_{2}, x_{2}+y\right)-\frac{2 x z}{y\left(y-x_{2}\right)}\left[N\left(y, x_{2}\right)\right.\right. \\
& \left.-N\left(y-x_{2}, y\right)+2 N\left(y-x_{2},-x_{2}\right)\right]+\frac{1}{x_{2}^{2}\left(y-x_{2}\right)}\left(x _ { 2 } \xi \left[O\left(y-x_{2}, y\right)\right.\right. \\
& \left.-N\left(y-x_{2}, y\right)\right]+\left(2 \xi y+y x_{2} z-y^{2} z-\xi x_{2}\right)\left[N\left(y, x_{2}\right)+O\left(y, x_{2}\right)\right] \\
& \left.\left.+y\left(z\left(y+x_{2}\right)-2 \xi\right)\left[N\left(y-x_{2},-x_{2}\right)+O\left(y-x_{2},-x_{2}\right)\right]\right)\right\} .
\end{aligned}
$$

The complete matching result is the sum of that in eq. (4.12) and that in eq. (4.26):

$$
\frac{1}{m_{A}} \Delta F_{q T}\left(x, \xi, k_{\perp}\right)=\left.\frac{1}{m_{A}} \Delta F_{q T}\left(x, \xi, k_{\perp}\right)\right|_{q \bar{q}+q G \bar{q}}+\left.\frac{1}{m_{A}} \Delta F_{q T}\left(x, \xi, k_{\perp}\right)\right|_{2 G+3 G} .
$$

\section{Summary}

SIDIS in target fragmentation region can be conveniently described with fracture functions, i.e., one can use QCD factorization with fracture functions to make predictions in this region. If the transverse momentum of the produced hadron is in the region $Q \gg k_{\perp} \gg \Lambda_{\mathrm{QCD}}$, the standard collinear factorization can also be used. Therefore, fracture functions can be factorized or matched with parton distributions and fragmentation functions. We have studied the matching up to twist-3 level. At the order of $\alpha_{s}$ considered in this work, fracture functions are only matched to twist-2 parton fragmentation functions with twist-2- or twist-3 parton distributions. There is no contribution from chirality-odd parton distributions. We have derived perturbative coefficient functions in the factorization or matching of twist-2and twist-3 fracture functions. Especially in the derivation of twist-3 fracture functions, we find that the results can be written in a gauge invariant form. These results will be useful for modeling of fracture functions and resummation of large logarithms of $k_{\perp}$ in collinear factorization. 


\section{Acknowledgments}

The work is supported by National Natural Science Foundation of China (No. 12075299, 11821505, 11935017 and 12047503) and by the Strategic Priority Research Program of Chinese Academy of Sciences, Grant No. XDB34000000. K.B. Chen is supported by National Natural Science Foundation of China (Nos. 11947055, 12005122) and Shandong Province Natural Science Foundation No. ZR2020QA082. X.B. Tong is supported by the CUHK-Shenzhen university development fund under Grant No. UDF01001859.

Open Access. This article is distributed under the terms of the Creative Commons Attribution License (CC-BY 4.0), which permits any use, distribution and reproduction in any medium, provided the original author(s) and source are credited.

\section{References}

[1] J.C. Collins, D.E. Soper and G.F. Sterman, Transverse Momentum Distribution in Drell-Yan Pair and $W$ and $Z$ Boson Production, Nucl. Phys. B 250 (1985) 199 [inSPIRE].

[2] J.C. Collins, D.E. Soper and G.F. Sterman, Factorization for Short Distance Hadron-Hadron Scattering, Nucl. Phys. B 261 (1985) 104 [inSPIRE].

[3] X.-d. Ji, J.-p. Ma and F. Yuan, QCD factorization for semi-inclusive deep-inelastic scattering at low transverse momentum, Phys. Rev. D 71 (2005) 034005 [hep-ph/0404183] [InSPIRE].

[4] X.-d. Ji, J.-P. Ma and F. Yuan, QCD factorization for spin-dependent cross sections in DIS and Drell-Yan processes at low transverse momentum, Phys. Lett. B 597 (2004) 299 [hep-ph/0405085] [INSPIRE].

[5] ZEUS collaboration, Observation of events with a large rapidity gap in deep inelastic scattering at HERA, Phys. Lett. B 315 (1993) 481 [INSPIRE].

[6] L. Trentadue and G. Veneziano, Fracture functions: An Improved description of inclusive hard processes in QCD, Phys. Lett. B 323 (1994) 201 [INSPIRE].

[7] D. Graudenz, One particle inclusive processes in deeply inelastic lepton-nucleon scattering, Nucl. Phys. B 432 (1994) 351 [hep-ph/9406274] [INSPIRE].

[8] A. Berera and D.E. Soper, Behavior of diffractive parton distribution functions, Phys. Rev. D 53 (1996) 6162 [hep-ph/9509239] [INSPIRE].

[9] J.C. Collins, Proof of factorization for diffractive hard scattering, Phys. Rev. D 57 (1998) 3051 [Erratum ibid. 61 (2000) 019902] [hep-ph/9709499] [INSPIRE].

[10] F.A. Ceccopieri and L. Trentadue, Semi-inclusive Drell-Yan Process as a Factorization Analyzer, Phys. Lett. B 668 (2008) 319 [arXiv:0805.3467] [INSPIRE].

[11] F.A. Ceccopieri, Associated production of one particle and a Drell-Yan pair in hadronic collisions, Phys. Lett. B 703 (2011) 491 [arXiv:1012.0507] [INSPIRE].

[12] J.C. Collins, L. Frankfurt and M. Strikman, Diffractive hard scattering with a coherent Pomeron, Phys. Lett. B 307 (1993) 161 [hep-ph/9212212] [INSPIRE].

[13] H1 collaboration, Measurement and QCD analysis of the diffractive deep-inelastic scattering cross-section at HERA, Eur. Phys. J. C 48 (2006) 715 [hep-ex/0606004] [inSPIRE]. 
[14] M. Goharipour, H. Khanpour and V. Guzey, First global next-to-leading order determination of diffractive parton distribution functions and their uncertainties within the xFitter framework, Eur. Phys. J. C 78 (2018) 309 [arXiv: 1802.01363] [InSPIRE].

[15] A. Accardi et al., Electron Ion Collider: The Next QCD Frontier: Understanding the glue that binds us all, Eur. Phys. J. A 52 (2016) 268 [arXiv:1212.1701] [InSPIRE].

[16] X. Cao et al., Electron ion collider in China, Nucl. Technol. 43 (2020) 20001.

[17] D.P. Anderle et al., Electron-ion collider in China, Front. Phys. (Beijing) 16 (2021) 64701 [arXiv:2102.09222] [INSPIRE].

[18] M. Anselmino, V. Barone and A. Kotzinian, SIDIS in the target fragmentation region: Polarized and transverse momentum dependent fracture functions, Phys. Lett. B 699 (2011) 108 [arXiv: 1102.4214] [INSPIRE].

[19] X.P. Chai, K.B. Chen, J.P. Ma and X.B. Tong, Fracture functions in different kinematic regions and their factorizations, JHEP 10 (2019) 285 [arXiv:1903.00809] [INSPIRE].

[20] M. Diehl and S. Sapeta, On the analysis of lepton scattering on longitudinally or transversely polarized protons, Eur. Phys. J. C 41 (2005) 515 [hep-ph/0503023] [INSPIRE].

[21] A. Bacchetta, M. Diehl, K. Goeke, A. Metz, P.J. Mulders and M. Schlegel, Semi-inclusive deep inelastic scattering at small transverse momentum, JHEP 02 (2007) 093 [hep-ph/0611265] [INSPIRE].

[22] A.P. Chen, J.P. Ma and G.P. Zhang, Twist-3 Contributions in Semi-Inclusive DIS with Transversely Polarized Target, Phys. Lett. B 754 (2016) 33 [arXiv:1505.03217] [InSPIRE].

[23] A.V. Efremov and O.V. Teryaev, On Spin Effects in Quantum Chromodynamics, Sov. J. Nucl. Phys. 36 (1982) 140 [INSPIRE].

[24] A.V. Efremov and O.V. Teryaev, QCD Asymmetry and Polarized Hadron Structure Functions, Phys. Lett. B 150 (1985) 383 [INSPIRE].

[25] J.-w. Qiu and G.F. Sterman, Single transverse spin asymmetries, Phys. Rev. Lett. 67 (1991) 2264 [INSPIRE].

[26] J.-w. Qiu and G.F. Sterman, Single transverse spin asymmetries in direct photon production, Nucl. Phys. B 378 (1992) 52 [inSPIRE].

[27] J.-w. Qiu and G.F. Sterman, Single transverse spin asymmetries in hadronic pion production, Phys. Rev. D 59 (1999) 014004 [hep-ph/9806356] [INSPIRE].

[28] J. Kodaira and K. Tanaka, Polarized structure functions in QCD, Prog. Theor. Phys. 101 (1999) 191 [hep-ph/9812449] [INSPIRE].

[29] J. Zhou, F. Yuan and Z.-T. Liang, Transverse momentum dependent quark distributions and polarized Drell-Yan processes, Phys. Rev. D 81 (2010) 054008 [arXiv:0909.2238] [INSPIRE].

[30] A.V. Belitsky and D. Mueller, Scale dependence of the chiral-odd twist-3 distributions $h_{L}(x)$ and e(x), Nucl. Phys. B 503 (1997) 279 [hep-ph/9702354] [INSPIRE].

[31] H. Eguchi, Y. Koike and K. Tanaka, Single Transverse Spin Asymmetry for Large- $p_{T}$ Pion Production in Semi-Inclusive Deep Inelastic Scattering, Nucl. Phys. B 752 (2006) 1 [hep-ph/0604003] [INSPIRE].

[32] X.-D. Ji and J. Osborne, An Analysis of the next-to-leading order corrections to the $g_{T}\left(=g_{1}+g_{s}\right)$ scaling function, Nucl. Phys. B 608 (2001) 235 [hep-ph/0102026] [InSPIRE]. 
[33] X.-D. Ji, Gluon correlations in the transversely polarized nucleon, Phys. Lett. B 289 (1992) 137 [INSPIRE].

[34] Y. Koike, K. Tanaka and S. Yoshida, Master Formula for the Three-Gluon Contribution to Single Spin Asymmetry in Semi-Inclusive Deep Inelastic Scattering, Phys. Rev. D 83 (2011) 114014 [arXiv: 1104.0798] [INSPIRE].

[35] H. Beppu, Y. Koike, K. Tanaka and S. Yoshida, Contribution of Twist-3 Multi-Gluon Correlation Functions to Single Spin Asymmetry in Semi-Inclusive Deep Inelastic Scattering, Phys. Rev. D 82 (2010) 054005 [arXiv: 1007.2034] [INSPIRE].

[36] H. Eguchi, Y. Koike and K. Tanaka, Twist-3 Formalism for Single Transverse Spin Asymmetry Reexamined: Semi-Inclusive Deep Inelastic Scattering, Nucl. Phys. B 763 (2007) 198 [hep-ph/0610314] [INSPIRE]. 